\title{
SYNTACTICAL TRANSFORMS
}

\section{BY \\ A. H. LIGHTSTONE AND A. ROBINSON(1)}

1. Introduction. A syntactical transform is a formal rule whose application to a sentence in a given formal language, in our case a variant of the lower predicate calculus, produces another sentence in the same language. For example, a set of instructions which converts a sentence into prenex normal form, or into Skolem's normal form, is a syntactical transform. The choice of the syntactical transforms which are considered in the present paper is motivated by mathematical considerations. We develop transforms which, in a sense to be made precise later, generalize the passage from the assertion of the existence of certain elements to the assertion that these elements are continuous functions of the parameters involved. We then show that, under certain conditions, the deducibility of an assertion of the former type from a given set of axioms entails the deducibility of its transform from a related set of axioms. Corresponding results for the concept of boundedness have been derived elsewhere [5].

Among the applications, we mention that if a sentence of a certain class holds in every completely divisible torsion-free abelian group then a transform of the sentence which expresses uniform continuity in the sense indicated above, holds in every completely divisible ordered abelian group. Also, if a sentence of a certain class holds in all groups then a transform of the sentence which expresses the topological continuity (in a weakened form) of any element whose existence is affirmed by the sentence, holds in every topological group. Finally, we show that in certain circumstances the mere existence of a solution of a system of equations entails a result concerning the continuity of the solution.

We shall make use of a particular many-sorted calculus (here called the uniform predicate calculus) in which no distinction is made between predicate variables and individual variables. Moreover, in certain cases we indicate a relation simply by the juxtaposition of its arguments. The use of this language proves convenient, though not essential, for our theory of syntactical transforms, which can also be formulated within the ordinary lower predicate calculus.

2. The uniform predicate calculus.

Symbols. Symbols fall into two categories-variables and functors. A

Received by the editors March 5, 1956.

(1) The work contained in this paper was incorporated in the Doctorate Thesis of A. H. Lightstone who wishes to gratefully acknowledge financial assistance received from the $\mathrm{Na}$ tional Research Council of Canada during the period 1953-1955. 
variable is a symbol $x_{i}^{k}$, where $k$ is a positive integer not exceeding $n$, while $i$ is any positive integer. The superscript denotes the class to which a variable belongs, while the subscript serves to differentiate between variables of the same class. Thus, a variable may be considered as an ordered pair of integers. In all, there are $n$ classes of variables. A functor is a symbol $\phi_{i}^{k}(\cdots)$ possessing a finite number of argument places each filled with a variable or functor. Again, the superscript denotes the class, while the subscript distinguishes between functors of the same class.

CONNECTIVES, QUANTIFIERS AND BRACKETS:

$$
\sim, \vee, A x_{i}^{m}, E x_{i}^{m},(,)
$$

where $m \in M, M$ a given set of positive integers not exceeding $n$, and $i$ is any positive integer.

WELL-FORMED FORMULAE. Wff are generated by a given set, called the generating set, of finite sequences of positive integers (not exceeding $n$ ) in the following manner: replace each integer in a sequence by any symbol with that integer as superscript; each of the resulting sequences of symbols is said to be a wff, and is called a generating wff. Furthermore, if $Q, B$ and $\mathcal{e}$ are any wff then so are

$$
\sim(Q), \quad(Q) \vee(\mathbb{B}), \quad A x_{i}^{m}(\mathcal{C}), \quad E x_{i}^{m}(\mathbb{e})
$$

where $m$ and $i$ are as above, provided no variable is both free in $a$ and bound in $B$ or vice versa, and provided $x_{i}^{m}$ is free in $\mathcal{C}$. The terms "free" and "bound" have the usual meaning. The usual convention for dropping brackets will be followed.

Provable formulae. In the following $Q, \Re, \mathfrak{C}$ are any wff; $m$ and $i$ are as above; $y$ denotes any symbol with superscript $m ; Q \supset \beta$ is an abbreviation for $\sim Q \vee B ;(Q)_{2}^{w}$ denotes the formula obtained from $Q$ by replacing each instance of $z$ in $a$ by $w$, and replacing each bound instance of $w$ in $a$ by $z$ where $w$ and $z$ denote symbols with the same superscript. We now say that the following are provable provided they are wff:

2.1. $a \vee Q \supset a$;

2.2. $a \supset Q \vee B$;

2.3. $a \vee B \supset B \vee a$;

2.4. $(a \supset B) \supset(\mathbb{e} \vee a \supset \mathfrak{e} \vee B)$;

2.5. $A x_{i}^{m}(Q) \supset(Q)_{x_{i}}^{y_{m}}$;

2.6. (Q) $)_{x_{i}}^{y_{m}} \supset E x_{i}^{m}(a)$.

Further, we say that

2.7. $Q$ is provable if $a$ is provable and $Q \supset Q$ is provable.

2.8. $Q \supset A x_{i}^{m}(B)$ is provable if $Q \supset \beta$ is provable and $x_{i}^{m}$ does not occur in $a$ but is free in $B$.

2.9. $E x_{i}^{m}(B) \supset Q$ is provable if $B \supset Q$ is provable and $x_{i}^{m}$ does not occur in $Q$ but is free in $B$. 


$$
A x_{i}^{0} A x_{j}^{h} \cdots A x_{s}^{r} E x_{p}^{k}\left((B)_{\phi_{t}^{k}\left(x_{i}^{0} x_{i}^{k} \cdots x_{i}^{r}\right)}^{x^{k}} \supset a\right.
$$

is provable if $A x_{i}^{o} A x_{j}^{h} \cdots A x_{s}^{r}(B) \supset Q$ is provable, providing $\phi_{t}^{k}\left(x_{i}^{o} x_{j}^{h} \cdots x_{s}^{r}\right)$ occurs in $B$ but not in $Q$, while $x_{p}^{k}$ does not occur in $B$.

It is readily shown that the Uniform Predicate Calculus is consistent, since we can show that not every wff is provable. In particular, it is easy to show that

$$
\sim(a \vee \sim a)
$$

is not provable.

We introduce the idea of a structure in which a wff can be interpreted in the usual way. A structure, $S$, is a set of constants together with a function, $f$, defined over certain finite sequences of the constants, and taking the value 0 or 1 . A wff is said to be defined in $S$ if we can correlate the variables and functors of the language with constants of $S$ in such a manner that the image under the correlation of each generating wff appearing in the given wff (and all other generating wff obtained from the same generating sequence) is defined under the function $f$. We say that a generating wff defined in the structure $S$ holds in $S$ if its image under the correlation takes the value 1 under $f$, and that it does not hold in $S$ if its image takes the value 0 . Furthermore, if $a$ and $B$ are any wff, we say that

$\sim Q$ holds in $S$ iff $Q$ does not hold in $S$;

$a \vee \otimes$ holds in $S$ iff $a$ holds in $S$ or $B$ holds in $S$;

$A x_{i}^{m}(Q)$ holds in $S$ iff $(Q)_{x_{i}^{m}}^{y_{m}}$ holds in $S$ no matter what symbol $y$ is, provided only that it have superscript $m$;

$E x_{i}^{m}(Q)$ holds in $S$ iff $(Q)_{x_{i}^{m}}^{y_{m}}$ holds in $S$ for some $y$, where $y$ denotes a symbol with superscript $m$;

provided that $Q \bigvee \otimes, A x_{i}^{m}(a), E x_{i}^{m}(a)$ are wff.

It is clear that if a wff is defined in a particular structure, then either it holds in that structure or it does not hold in that structure, and that both cases cannot occur simultaneously.

We can now show that the Uniform Predicate Calculus is complete in the sense that a wff is provable if it holds in every structure in which it is defined. It is enough to show that every consistent (noncontradictory) set of wff holds in some structure. Now, if a set, $K$, of wff in prenex normal form is consistent, so is the set, $K^{*}$, of wff obtained from the wff of $K$ by replacing all existentially quantified variables by functors of the preceding universally quantified variables. Furthermore, $K$ holds in any structure in which $K^{*}$ holds. We now form the set $K^{* *}$ of wff obtained from those of $K^{*}$ by replacing each quantified variable in a matrix by a symbol with the same superscriptin all possible ways-and discarding each prefix. But if $K^{*}$ is consistent so is $K^{* *}$, and $K^{*}$ holds in every structure in which $K^{* *}$ holds. However, it can 
be shown (e.g. [4]) that any consistent set of wff free of quantifiers holds in some structure. Thus, any consistent set of wff holds in some structure.

We note that the lower Predicate Calculus is a special case of the Uniform Predicate Calculus. Interpret $x_{i}^{1}$ as individual variables, and $x_{i}^{k}$ as predicate variables of order $k-2$ for $2 \leqq k \leqq n$. Set $M=\{1\}$, and let the generating set consist of the sequences $(k, 1,1, \cdots, 1)$ with $2 \leqq k \leqq n$, where $k$ is followed by $k-21$ 's.

An extended predicate calculus results if we replace $M$ in the above interpretation by the set of all positive integers not exceeding $n$.

Furthermore, a many-sorted calculus is obtained by restricting the first $k$ classes to individual variables of various sorts, while the remaining classes represent relations (e.g. [6]); hence, $M=\{1,2, \cdots, k\}$.

In the following, we interpret the Uniform Predicate Calculus as a manysorted calculus in which, generally, relations are indicated by the juxtaposition of their arguments. Thus, generally, variables with different superscripts denote individual variables of different sorts.

3. Duplication. Let $Y$ be any formula, we denote by $D_{m}(Y)$ the formula obtained by applying the following syntactical transformation to $Y$.

3.1. Each variable $x_{j}^{m}$ or functor $\phi_{j}^{m}\left(a_{1} \cdots a_{n}\right)$ with superscript $m$ is replaced by $x_{2 j}^{m} x_{2 j+1}^{m}$ or $\phi_{2 j}^{m}\left(D_{m}\left(a_{1} \cdots a_{n}\right)\right) \phi_{2 j+1}^{m}\left(D_{m}\left(a_{1} \cdots a_{n}\right)\right)$ respectively; for example, $x_{i}^{p} x_{j}^{m} x_{k}^{r} \phi_{s}^{m}\left(x_{j}^{m} x_{k}^{r}\right)$ is replaced by

$$
\underset{x_{i} x_{2 j} x_{2 j+1}^{m}}{m} \underset{x_{k} \phi_{2 s}}{m}\left(x_{2 j}^{m} x_{2 j+1}^{m} \stackrel{r}{x_{k}}\right) \phi_{2 s+1}^{m}\left(x_{2 j}^{m} x_{2 j+1}^{m} x_{k}^{r}\right) .
$$

3.2. Each quantifier $A x_{j}^{m}$ or $E x_{j}^{m}$ with superscript $m$ is replaced by $A x_{2 j}^{m} A x_{2 j+1}^{m}$ or $E x_{2 j}^{m} E x_{2 j+1}^{m}$ respectively. In the following we denote $2 i$ by $i_{1}$, $2 i+1$ by $i_{2}$, and the components of $D_{m}(y)$ by $y_{1}$ and $y_{2}$ if $y$ is a function; thus, if $y$ is $\phi_{s}^{m}\left(x_{j}^{m} x_{k}^{r}\right)$ then $y_{1}$ is $\phi_{2 s}^{m}\left(x_{2 j}^{m} x_{2 j+1}^{m} x_{k}^{r}\right)$ and $y_{2}$ is $\phi_{2 s+1}^{m}\left(x_{2 j}^{m} x_{2 j+1}^{m} x_{k}^{r}\right)$. We note that $D_{m}\left(Q_{x_{i}}^{y_{m}}\right)$ is $\left(\left(D_{m}(Q)\right)_{x_{i_{1}}}^{y_{1}}\right)_{x_{i_{2}}}^{y_{2}}$ which we write simply as $\left(D_{m}(Q)\right)_{x_{i_{1}}}^{y_{1} x_{i_{2}}} x_{i_{2}}$.

We shall show that $D_{m}^{2}(Y)$ is provable provided it is a wff and $Y$ is provable. (Note that a generating sequence when duplicated may not be a generating sequence; thus, $D_{m}(Y)$ is not always a wff.) We use induction on the order of a provable wff where the order is the minimum number of applications of rules of inference required to establish provability of the wff. Thus, the wff 2.1-2.6 have order zero; we show that their duplicates are provable. This is clearly true for $2.1-2.4$; we consider 2.5 . Now,

$$
A x_{i_{1}}^{m} A x_{i_{2}}^{m}\left(D_{m}(Q)\right) \supset\left(A x_{i_{2}}^{m}\left(D_{m}(Q)\right)\right)_{x_{i} m}^{y_{1}} \text { is provable }
$$

but,

$$
\left(A x_{i_{2}}^{m}\left(D_{m}(Q)\right)\right)_{x_{i_{1}} m}^{y_{1}} \supset\left(D_{m}(Q)\right)_{x_{i_{1} m} x_{i_{2} m}}^{\nu_{1} y_{2}} \text { is provable, }
$$

thus, 


$$
A x_{i_{1}}^{m} A x_{i_{2}}^{m}\left(D_{m}(Q)\right) \supset\left(D_{m}(Q)\right)_{x_{i_{1}} x_{i_{2}} m}^{y_{1} y_{2}} \text { is provable. }
$$

The duplicate of 2.6 can be shown to be provable in a similar way. Thus, any provable wff of order zero has a provable transform. Suppose that any provable wff of order $<n$ has a provable transform; we shall show that then any provable wff with order $n$ has a provable transform. We show, in fact, that the duplicate of a wff is provable if the wff is obtained by applying a rule of inference to a provable wff of order $<n$.

Let $Q$ and $Q \supset Q$ be provable wff of order $<n$; then $D_{m}(Q)$ and $D_{m}(Q \supset \beta)$ are provable. But $D_{m}(Q \supset \Theta)$ is $D_{m}(Q) \supset D_{m}(\beta)$; hence, by $2.7, D_{m}(\beta)$ is provable.

Let $Q \supset \otimes$ be provable of order $<n$, where $x_{i}^{m}$ is free in $\&$ but does not occur in $Q$; then $D_{m}(Q \supset Q)$ is provable, i.e. $D_{m}(Q) \supset D_{m}(Q)$ is provable where $x_{i_{1}}^{m}$ and $x_{i_{2}}^{m}$ are free in $D_{m}(Q)$ but do not occur in $D_{m}(Q)$. Hence, by $2.8, D_{m}(Q)$ $\supset A x_{i_{1}}^{m} A x_{i_{2}}^{m}\left(D_{m}(B)\right)$ is provable.

It can be shown similarly that the duplicate of a wff is provable if the wff is obtained by applying 2.9 to a provable wff of order $<n$.

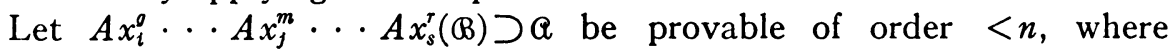
$\phi_{t}^{k}\left(x_{i}^{g} \cdots x_{j}^{m} \cdots x_{s}^{r}\right)$ occurs in $@$ but not in $Q$, and $x_{p}^{k}$ does not occur in $\Theta$. Then, $A x_{i}^{o} \cdots A x_{j_{1}}^{m} A x_{j_{2}}^{m} \cdots A x_{s}^{\tau}\left(D_{m}(B)\right) \supset D_{m}(Q)$ is provable, where $\phi_{t}^{k}\left(x_{i}^{o} \cdots x_{j_{1}}^{m} x_{j_{2}}^{m} \cdots x_{s}^{\tau}\right)$ occurs in $D_{m}(B)$ but not in $D_{m}(Q)$, and $x_{p}^{k}$ does not occur in $D_{m}(B)$; thus,

$$
A x_{i}^{o} \cdots A x_{j_{1}}^{m} A x_{j_{2}}^{m} \cdots A x_{s}^{r} E x_{p}^{k}\left(\left(D_{m}(B)\right)_{\phi_{l}^{k}\left(x_{1}^{o} \cdots x_{i_{i}}^{m} x_{i}^{m}\right.}^{x_{i}^{k}} \cdots x_{i}^{r}\right), \supset D_{m}(Q)
$$

is provable by 2.10 .

Let $A x_{i}^{o} \cdots A x_{s}^{r}(\beta) \supset Q$ be provable of order $<n$, where $\phi_{l}^{m}\left(x_{i}^{\sigma} \cdots x_{s}^{r}\right)$ occurs in $B$ but not in $Q$, and $x_{p}^{m}$ does not occur in $Q$. Then $A x_{i}^{a} \cdots$ $A x_{s}^{r}\left(D_{m}(B)\right) \supset D_{m}(Q)$ is provable where neither $\phi_{l_{1}}^{m}\left(x_{i}^{q} \cdots x_{s}^{r}\right)$ nor $\phi_{t_{2}}^{m}\left(x_{i}^{q} \cdots x_{s}^{r}\right)$ occurs in $D_{m}(Q)$, while both occur in $D_{m}(B)$, and neither $x_{p_{1}}^{m}$ nor $x_{p_{2}}^{m}$ occurs in $D_{m}(B)$. Hence,

$$
A x_{i}^{g} \cdots A x_{s}^{r} E x_{p_{1}}^{m} E x_{p_{2}}^{m}\left(\left(D_{m}(\&)\right)_{\left.\phi_{t_{1}}^{m} x_{i}^{p_{1}} \cdots x_{s}^{r}\right) \phi_{t_{2}}^{m}\left(x_{i}^{p_{2}} \cdots x_{*}^{r}\right)}^{x^{m}} \supset D_{m}(Q)\right.
$$

is provable, by 2.10 .

We have now shown that every provable wff of order $n$ has a provable transform provided that every provable wff of order $<n$ has a provable transform. This completes the induction; thus, if $Y$ is provable, so is $D_{m}(Y)$.

Of course, $D_{m}(Y)$ may itself be duplicated with respect to another class, say $k$. Thus, if $Y$ is provable so is $D_{k}\left(D_{m}(Y)\right)$. But it is clear that $D_{k}\left(D_{m}(Y)\right)$ is precisely $D_{m}\left(D_{k}(Y)\right)$; i.e. the order of duplication does not affect the result. Hence, if a provable wff is duplicated simultaneously with respect to two classes, the resulting formula is provable (provided that it is a wff). Finally, we see that if a provable wff is duplicated simultaneously with respect to all 
classes occurring in the wff, then the resulting formula is provable.

We have demonstrated that if $Y$ is a provable wff, then so is $D(Y)$, where $D(Y)$ is obtained from $Y$ by applying the following syntactical transformation:

3.3. Each variable $x_{j}^{t}$ or functor $\phi_{j}^{t}$ is replaced by $x_{2 j}^{t} x_{2 j+1}^{t}$ or $\phi_{2 j}^{i} \phi_{2 j+1}^{t}$, respectively, $t=1,2, \cdots, n, j=1,2, \cdots$; for example, $x_{i}^{p} \phi_{s}^{m}\left(x_{j}^{m} x_{t}^{\tau}\right)$ is replaced by

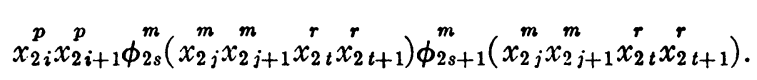

3.4. Each quantifier $A x_{j}^{t}$ or $E x_{j}^{t}$ is replaced by $A x_{2 j}^{t} A x_{2 j+1}^{t}$ or $E x_{2 j}^{t} E x_{2 j+1}^{t}$ respectively.

4. Relativization of quantifiers. In this section we confine ourselves to that portion of our language which contains no functors. There is no loss in generality in doing so, since a functor, $\phi_{i}^{k}\left(x_{1}^{m} \cdots x_{n}^{p}\right)$, with $n$ argument places can always be replaced by the wff $x_{i}^{r} x_{1}^{m} \cdots x_{n}^{p} x_{j}^{k}$ which is interpreted as $x_{j}^{k}=x_{i}^{r} x_{1}^{m} \cdots x_{n}^{p}$, where $x_{i}^{r}$ plays the role of $\phi_{i}^{k}[3]$ with the appropriate axioms.

We consider now a syntactical transform which has made several appearances in the literature (e.g. [1]). The purpose of this transform is to restrict the range of a variable being quantified to a set of variables satisfying a given wff. The transform is as follows:

4.1. Replace $A x_{i}^{m}(Q)$ by $A x_{i}^{m}\left(x_{i}^{m} R_{m} \supset Q_{m}\right)$.

4.2. Replace $E x_{i}^{m}(Q)$ by $E x_{i}^{m}\left(x_{i}^{m} R_{m} \wedge Q_{m}\right)$

where $Q \wedge \cap$ is an abbreviation for $\sim(\sim Q \vee \sim Q)$. Here, $m$ is fixed, and $R_{m}$ denotes a fixed sequence of variables each with superscript different from $m$, such that $x_{i}^{m} R_{m}$ is a wff; we call $R_{m}$ the relativizing sequence of the transform. We denote by $Y_{m}$ the wff obtained by applying the transform to $Y$. Clearly, if $Y$ is quantifier free then $Y_{m}$ is $Y$.

It can be shown (compare [1]) that

$$
\operatorname{Ex}_{j}^{m}\left(x_{i}{ }_{i} R_{m}\right) \supset Y_{m}
$$

is provable, provided that $Y$ is provable, has no free variables with superscript $m$, and no variable that occurs in $R_{m}$ is bound in $Y$.

We shall say that a set of wff, $S_{K}$, is a supporting set of a transform, $K$, if the $K$-transform of any provable wff is deducible from $S_{K}$. Thus, if we restrict the above transform to wff which possess no free variables with superscript $m$ and no bound variables that occur in $R_{m}$, then $E x_{j}^{m}\left(x_{j}^{m} R_{m}\right)$ is a supporting set of the transform.

So far we have relativized with respect to just one class of symbols. We now introduce another relativizing sequence, $R_{k}$, and relativize quantified variables with superscript $k$. Thus,

$$
A x_{i}^{k}(Q) \text { is replaced by } A x_{i}^{k}\left(x_{i}^{k} R_{k} \supset \mathfrak{Q}_{k}\right)
$$




$$
E x_{i}^{k}(Q) \text { is replaced by } E x_{i}^{k}\left(x_{i}^{k} R_{k} \wedge Q_{k}\right) \text {. }
$$

Again, $R_{k}$ is a sequence of variables each with superscript different from $k$. It follows that if $Y$ is provable, has no free variables with superscript $m$ or $k$, and if no variable that occurs in $R_{m}$ or $R_{k}$ is bound in $Y$, then

$$
E x_{j}^{k}\left(x_{j}^{k} R_{k}\right) \supset\left(E x_{j}^{m}\left(x_{j}^{m} R_{m}\right) \supset Y_{m}\right)_{k} \quad \text { is provable. }
$$

But, $\left(Y_{m}\right)_{k}$ is $\left(Y_{k}\right)_{m}$; accordingly, we denote $\left(Y_{m}\right)_{k}$ by $Y_{m k}$. Thus,

$$
\left(E x_{j}^{m}\left(x_{j}^{m} R_{m}\right) \supset Y_{m}\right)_{k}
$$

is $E x_{j}^{m}\left(x_{j}^{m} R_{m}\right) \supset Y_{m k}$; hence, $E x_{j}^{k}\left(x_{j}^{k} R_{k}\right) \wedge E x_{j}^{m}\left(x_{j}^{m} R_{m}\right) \supset Y_{m k}$ is provable.

Generally, if $Y$ is provable, has no free variables, and no variable bound in $Y$ occurs in any $R_{i}, i=1,2, \cdots, n$, then $E x_{j}^{1}\left(x_{j}^{1} R_{1}\right) \wedge E x_{j}^{2}\left(x_{j}^{2} R_{2}\right) \wedge \cdots$ $\wedge E x_{j}^{n}\left(x_{j}^{n} R_{n}\right) \supset R(Y)$ is provable, where $R(Y)$ denotes the wff obtained by relativizing $Y$ with respect to all classes. Thus, a supporting set, $S_{R}$, of the $R$ transform is constituted by

$$
E x_{j}^{1}\left(x_{j}^{1} R_{1}\right), E x_{j}^{2}\left(x_{j}^{2} R_{2}\right), \cdots, E x_{j}^{n}\left(x_{j}^{n} R_{n}\right) .
$$

5. The continuity at zero transform. We introduce now a transform which generalizes the idea of continuity at zero for a function which takes the value zero when its arguments are zero. A wff in prenex normal form is transformed by relativizing its matrix with respect to the variables in the prefix, and quantifying any variables introduced in the relativizing sequences. This transform is defined only for wff in prenex normal form in which no more than one class of variables is quantified, and which contains no free variables of a quantified class; we shall say that a wff belongs to the class $y$ if it satisfies these restrictions. The transform is as follows:

5.1. Adjoin to the left of the prefix the sequence of quantifiers obtained as follows:

(a) write down the prefix in reverse order, interchanging $A$ and $E$,

(b) remove all but the last of each sequence of universal or existential quantifiers,

(c) change all superscripts to those of another class (not mentioned previously.

5.2. Relativize with respect to the class quantified in the given wff, the relativizing sequence for each quantified variable being the variable introduced into the prefix which corresponds to the block of universal or existential quantifiers to which the quantifier concerned belongs.

For example, the transform of

$$
E x_{i}^{m} \operatorname{Ex}_{j}^{m} A x_{k}^{m}(Q)
$$




$$
E x_{k}^{r} A x_{i}^{r} E x_{i}^{m} E x_{j}^{m} A x_{k}^{m}\left(x_{i}^{m} x_{i}^{r} \wedge\left(x_{j}^{m} x_{i}^{r} \wedge\left(x_{k}^{m} x_{k}^{r} \supset Q\right)\right)\right) \text {. }
$$

We shall denote the transform of $Y$, as above, by $Z(Y)$.

If in the interpretation the wff $x_{i}^{m} x_{j}^{r}$ means that $x_{i}^{m}$ is small, then the transform of $A x_{i}^{m} E x_{j}^{m}(a)$, namely,

$$
A x_{j}^{r} E x_{i}^{r} A x_{i}^{m} E x_{j}^{m}\left(x_{i}^{m} x_{i}^{r} \supset\left(x_{i}^{m} x_{i}^{r} \wedge Q\right)\right)
$$

expresses the continuity at zero of the functor satisfying $Q$. For this reason we call this transform the "continuity at zero" transform.

A related transform is considered by one of the authors in [5]; this transform is termed the "bounding" transform, since under an appropriate interpretation the boundedness of a functor can be obtained from its existence by use of the transform. The bounding transform differs from the continuity transform only in its treatment of the prefix of a wff; for example, if the prefix is

$$
A x_{i}^{m} \cdots E x_{j}^{m} \cdots A x_{k}^{m} \cdots E x_{s}^{m}
$$

where only the initial quantifier in each block is shown, then the transformed prefix is

$$
A x_{i}^{r} E x_{j}^{r} A x_{k}^{r} E x_{s}^{r} A x_{i}^{m} \cdots E x_{j}^{m} \cdots A x_{k}^{m} \cdots E x_{s}^{m} .
$$

It is shown that a supporting set of the bounding transform consists of the following:

$$
E x_{i}^{r} E x_{j}^{m}\left(x_{j}^{m} x_{i}^{r}\right)
$$

$$
A x_{i}^{r} A x_{j}^{r}\left(x_{i}^{r} x_{j}^{r} \vee x_{j}^{r} x_{i}^{r}\right) \text {; }
$$

$$
A x_{i}^{r} A x_{j}^{r} A x_{k}^{r}\left(x_{i}^{r} x_{j}^{r} \wedge x_{j}^{r} x_{k}^{r} \supset x_{i}^{r} x_{k}^{r}\right) \text {; }
$$

$$
A x_{i}^{m} A x_{j}^{r} A x_{k}^{r}\left(x_{i}^{m} x_{j}^{r} \wedge x_{j}^{r} x_{k}^{r} \supset x_{i}^{m} x_{k}^{r}\right) \text {; }
$$

where the transform is restricted to wff belonging to the class $Z$ consisting of those members of the class $Y$ whose prefix does not have the form

$$
\cdots A \cdots E \cdots A \cdots
$$

where the dots denote quantifiers. It is also shown that if 5.3 is replaced by

$$
A x_{i}^{r} E x_{j}^{m}\left(x_{j}^{m} x_{i}^{r}\right)
$$

then the bounding transform may be applied to wff possessing free variables of the class $m$.

We shall show that if the continuity transform is applied only to wff belonging to the class $Z$, then 5.4-5.7 constitute a supporting set of this transform. We denote this set by $S_{z}$. First, we require the following 
5.8. ThEOREM. If $Y \in \mathcal{Y}$ and has the form

$$
A x_{i_{1}}^{m} \cdots A x_{i_{s}}^{m} E x_{j_{1}}^{m} \cdots E x_{i_{t}}^{m} A x_{k_{1}}^{m} \cdots A x_{k_{u}}^{m}\left(B \left(x_{i_{1}}^{m} \cdots x_{i_{s}}^{m}\right.\right.
$$

$$
\left.\left.x_{j_{1}}^{m} \cdots x_{j_{t}}^{m}, x_{k_{1}}^{m} \cdots x_{k_{u}}^{m}\right)\right)
$$

then $Y$ is consistent provided $\{Z(Y)\} \cup S_{Z}$ is consistent.

Proof. We suppose we are given a structure $S^{*}$ such that $\{Z(Y)\} \cup S_{Z}$ holds in $S^{*}$. Hence,

$$
\begin{aligned}
& a_{1} h(y) \wedge \cdots \wedge a_{s} h(y) \supset f_{1}\left(y, a_{1} \cdots a_{s}\right) y \wedge \cdots \wedge f_{t}\left(y, a_{1} \cdots a_{s}\right) y \\
& \wedge\left(b _ { 1 } z \wedge \cdots \wedge b _ { u } z \supset \otimes \left(a_{1} \cdots a_{s}, f_{1}\left(y, a_{1} \cdots a_{s}\right) \cdots f_{t}\left(y, a_{1} \cdots a_{s}\right),\right.\right. \\
& \left.\left.b_{1} \cdots b_{u}\right)\right)
\end{aligned}
$$

holds in $S^{*}$ for any choice of $a_{1} \cdots a_{s}, b_{1} \cdots b_{u}, y$ where $h, f_{1} \cdots f_{t}$ are given functions over $S^{*}$, and $z$ is a given constant. Note that $a_{1} \cdots a_{s}$, $b_{1} \cdots b_{u}$ are correlated with variables with superscript $m$, while $y$ and $z$ are correlated with variables having superscript $r$.

We shall construct a structure $S$ in which 5.9 holds. The constants of $S$ are obtained from those of $S^{*}$ and $t$ symbols $F_{1}\left(x_{1} \cdots x_{8}\right), \cdots, F_{t}\left(x_{1} \cdots x_{8}\right)$ each having $s$ argument places. First, we say that a constant of $S^{*}$ has order zero in $S$; next, we say that $F_{i}\left(x_{1} \cdots x_{s}\right), i=1, \cdots, t$, is a constant of $S$ of order $n$ if its arguments are constants of $S$ of order $<n$, and at least one argument has order $n-1$. We denote the constants of $S$ by

$$
c_{1}, c_{2}, \cdots, c_{n}, \cdots
$$

such that $c_{i}$ precedes $c_{j}$ if the order of $c_{i}$ is not greater than the order of $c_{j}$.

Having defined the constants of $S$ it remains to define for each generating wff of $B$ whether or not it holds in $S$ when its symbols are replaced by constants of $S$. When this is done we will have constructed a structure in which 5.9 is defined.

Let $W$ be the set of all wff obtained from the generating wff of $B$ by replacing symbols by constants of $S$ in all possible ways. Let $W_{k}$ be the set of all wff obtained in this manner by using only constants of $S$ of order $\leqq k$; then

$$
W_{0} \subset W_{1} \subset W_{2} \subset \cdots \text { and } \quad \sum_{i} W_{i}=W .
$$

It is known that if the wff of each $W_{k}$ are assigned values in such a manner that 5.9 holds when the universally quantified variables are restricted to constants of order $<k$, then a valuation of $W$ can be found such that 5.9 holds in $S$.

We show now how to assign values to the wff of $W_{k}$ so that the above requirement is obtained. First, we define two mappings of the constants of $S$ 
of order $\leqq k$ into the constants of $S^{*}$ as follows:

$$
\mu c_{m}=\left\{\begin{array}{l}
z, \text { if } c_{m} \text { has order } k ; \\
\text { minimum }\left(\mu F_{1}\left(c_{m} \cdots c_{m}\right), h\left(\mu F_{1}\left(c_{m} \cdots c_{m}\right)\right)\right), \text { otherwise }
\end{array}\right.
$$

where minimum $\left(a_{i}, a_{j}\right)=a_{i}$ if $a_{i} a_{j}$ holds in $S^{*}$; otherwise minimum $\left(a_{i}, a_{j}\right)$ $=a_{j}$.

$\lambda c_{m}=a_{i}$, if $c_{m}$ has order zero, where $a_{i} \mu c_{m}$ holds in $S^{*}$

$$
\lambda F_{i}\left(c_{j_{1}} \cdots c_{j_{s}}\right)=f_{i}\left(\mu F_{1}\left(c_{j_{1}} \cdots c_{j_{s}}\right), \lambda c_{j_{1}} \cdots \lambda c_{j_{s}}\right) \text {. }
$$

We show that $\mu c_{m}=\mu c_{n}$ if $c_{m}$ and $c_{n}$ have the same order: true if $c_{m}$ has order $k$; suppose true if $c_{m}$ has order $>j$. Let $c_{p}$ and $c_{q}$ have order $j$; then

$$
\mu c_{p}=\operatorname{minimum}\left(\mu F_{1}\left(c_{p} \cdots c_{p}\right), h\left(\mu F_{1}\left(c_{p} \cdots c_{p}\right)\right)\right)
$$

but $F_{1}\left(c_{p} \cdots c_{p}\right)$ has order $>j$, and

$$
\operatorname{order} F_{1}\left(c_{p} \cdots c_{p}\right)=\operatorname{order} F_{1}\left(c_{q} \cdots c_{q}\right)
$$

thus, by the induction assumption, $\mu F_{1}\left(c_{p} \cdots c_{p}\right)=\mu F_{1}\left(c_{q} \cdots c_{q}\right)$, hence, $\mu c_{p}=$ minimum $\left(\mu F_{1}\left(c_{q} \cdots c_{q}\right), h\left(\mu F_{1}\left(c_{q} \cdots c_{q}\right)\right)\right)=\mu c_{q}$.

It is now clear that $\mu c_{m} \mu c_{n}$ holds if order $c_{m} \leqq \operatorname{order} c_{n}$, since $\mu c_{m} \mu F_{1}\left(c_{m} \cdots c_{m}\right)$ holds; thus, $\mu c_{m} \mu c_{n}$ holds if order $c_{n}=1+\operatorname{order} c_{m}$, but $\mu c_{n} \mu c_{p}$ holds if order $c_{p}=1+\operatorname{order} c_{n}$, hence $\mu c_{m} \mu c_{p}$ holds if order $c_{p}=2+\operatorname{order} c_{m}$; carrying this on we see that $\mu c_{m} \mu c_{n}$ holds if order $c_{m} \leqq \operatorname{order} c_{n}$.

We show now that $\lambda c_{m} \mu c_{m}$ holds in $S^{*}$ : true if $c_{m}$ has order zero; suppose true if $c_{m}$ has order $<n$. Let $c_{q}=F_{i}\left(c_{j_{1}} \quad \cdot c_{j_{s}}\right)$ have order $n$, then $\lambda c_{j_{1}} \mu c_{j_{1}} \wedge \cdots$ $\wedge \lambda c_{j_{s}} \mu c_{j_{\text {s }}}$ holds; hence, by 5.6, $\lambda c_{j_{1}} \mu c_{j_{\nu}} \wedge \cdots \wedge \lambda c_{j_{s}} \mu c_{j_{\nu}}$ holds, where $c_{j_{\nu}}$ has the maximum order of $c_{j_{1}}, \cdots, c_{j_{s}}$. But $\mu c_{j_{\nu}} h\left(\mu F_{1}\left(c_{j_{\nu}} \cdots c_{j_{\nu}}\right)\right)$ holds, hence, $\mu c_{j_{\nu}} h\left(\mu F_{i}\left(c_{j_{1}} \cdots c_{j_{s}}\right)\right)$ holds, since

$$
\mu F_{1}\left(c_{j_{1}} \cdots c_{j_{\nu}}\right)=\mu F_{i}\left(c_{j_{1}} \cdots c_{j_{s}}\right) ;
$$

thus, by 5.6, $\lambda c_{j_{1}} h\left(\mu c_{q}\right) \wedge \cdots \wedge \lambda c_{j_{s}} h\left(\mu c_{q}\right)$ holds; hence, from 5.10, $f_{i}\left(\mu F_{1}\left(c_{j_{1}} \cdots c_{j_{s}}\right), \lambda c_{j_{1}} \cdots \lambda c_{j_{s}}\right) \mu c_{q}$ holds, then, $\lambda F_{i}\left(c_{j_{1}} \cdots c_{j_{s}}\right) \mu c_{q}$ holds, i.e. $\lambda c_{q} \mu c_{q}$ holds.

Now, in 5.10, set $a_{1}=\lambda c_{j_{1}}, \cdots, a_{s}=\lambda c_{j_{s}} ; b_{1}=\lambda c_{k_{1}}, \cdots, b_{u}=\lambda c_{k_{u}} ; y$ $=\mu F_{1}\left(c_{j_{1}} \cdots c_{j_{s}}\right)$. But $\lambda c_{j_{1}} h\left(\mu F_{1}\left(c_{j_{1}} \cdots c_{j_{s}}\right)\right) \wedge \cdots \wedge \lambda c_{j_{s}} h\left(\mu F_{1}\left(c_{j_{1}} \cdots c_{j_{s}}\right)\right)$ holds in $S^{*}$; and $\lambda c_{k_{1}} z \wedge \cdots \wedge \lambda c_{k_{u}} z$ holds in $S^{*}$, since $\lambda c_{k_{1}} \mu c_{k_{1}} \wedge \cdots \wedge \lambda c_{k_{u}} \mu c_{k_{u}}$ holds, and $\mu c_{i} z$ holds for any $i$. Hence, $B\left(\lambda c_{j_{1}} \cdots \lambda c_{j_{s}}, f_{1}\left(\mu F_{1}\left(c_{j_{1}} \cdots c_{j_{s}}\right)\right.\right.$, $\left.\left.\lambda c_{j_{1}} \cdots \lambda c_{j_{s}}\right) \cdots f_{t}\left(\mu F_{1}\left(c_{j_{1}} \cdots c_{j_{s}}\right), \lambda c_{j_{1}} \cdots \lambda c_{j_{s}}\right), \lambda c_{k_{1}} \cdots \lambda c_{k_{u}}\right)$ holds in $S^{*}$, i.e. $B\left(\lambda c_{j_{1}} \cdots \lambda c_{j_{s}}, \lambda F_{1}\left(c_{j_{1}} \cdots c_{j_{s}}\right) \cdots \lambda F_{t}\left(c_{j_{1}} \cdots c_{j_{s}}\right), \lambda c_{k_{1}} \cdots \lambda c_{k_{u}}\right)$ holds in $S^{*}$.

We now define the value in $S$ of any generating wff $c_{i_{1}} \cdots c_{i_{m}}$ to be the value in $S^{*}$ of the corresponding sequence $\lambda c_{i_{1}} \cdots \lambda c_{i_{m}}$ under the $\lambda$ mapping. Hence, it is clear that. 


$$
B\left(c_{j_{1}} \cdots c_{i_{s}}, F_{1}\left(c_{j_{1}} \cdots c_{j_{s}}\right) \cdots F_{t}\left(c_{j_{1}} \cdots c_{j_{s}}\right), c_{k_{1}} \cdots c_{k_{\mathrm{s}}}\right)
$$

holds in $S$ if $c_{j_{1}} \cdots c_{j_{d}}, c_{k_{1}} \cdots c_{k_{u}}$ are constants of order $<k$. We now define $t$ functions, $g_{1}\left(x_{1} \cdots x_{8}\right), \cdots, g_{t}\left(x_{1} \cdots x_{s}\right)$, in $S$ as follows: $g_{i}\left(c_{j_{1}} \cdots c_{j_{s}}\right)$ takes as its functional value the constants $F_{i}\left(c_{j_{1}} \cdots c_{j_{2}}\right)$. Thus, 5.9 , holds in $S$ when the universally quantified variables are restricted to constants of order $<k$.

Counter-example. We show that 5.8 does not apply generally; in particular, we show that there is a wff with prefix $E x_{i}^{m} A x_{j}^{m} E x_{k}^{m}$ such that 5.8 is false. Consider $Y=E x_{i}^{m} A x_{j}^{m} E x_{k}^{m}\left(x_{i}^{p} x_{i}^{m} x_{j}^{m} \wedge \sim x_{i}^{p} x_{i}^{m} x_{k}^{m}\right)$. Now,

$$
\sim A x_{j}^{m}\left(x_{t}^{p} x_{i}^{m} x_{j}^{m}\right) \vee A x_{j}^{m}\left(x_{i}^{p} x_{i}^{m} x_{j}^{m}\right) \text { holds in any structure, }
$$

i.e.

$$
E x_{i}^{m}\left(\sim x_{i}^{p} x_{i}^{m} x_{j}^{m}\right) \vee A x_{k}^{m}\left(x_{i}^{p} x_{i}^{m} x_{k}^{m}\right) \text { holds in any structure; }
$$

thus,

$$
E x_{i}^{m} A x_{k}^{m}\left(\sim x_{t}^{p} x_{i}^{m} x_{j}^{m} \vee x_{t}^{p} x_{i}^{m} x_{k}^{m}\right) \text { holds in any structure; }
$$

hence,

$$
A x_{i}^{m} E x_{j}^{m} A x_{k}^{m}\left(\sim x_{t}^{p} x_{i}^{m} x_{j}^{m} \vee x_{i}^{p} x_{i}^{m} x_{k}^{m}\right) \text { holds in any structure, }
$$

thus,

$$
E x_{i}^{m} A x_{j}^{m} E x_{k}^{m}\left(x_{i}^{p} x_{i}^{m} x_{j}^{m} \wedge \sim x_{i}^{p} x_{i}^{m} x_{k}^{m}\right) \text { is contradictory. }
$$

We shall show that $\{Z(Y)\} \cup S_{Z}$ is satisfied in some structure. Consider the structure $M$ with two classes of constants, $y_{k}^{j}$ and $d_{i}$, where $i, j, k$ $=\cdots,-2,-1,0,1,2, \cdots ;$ and a constant $x_{i}^{p}$.

We define:

$$
\begin{gathered}
\begin{array}{c}
y_{k}^{j} d_{i} \text { holds in } M \text { iff } j \leqq i ; \\
d_{i} d_{j} \text { holds in } M \text { iff } i \leqq j ; \\
x_{t}^{p} y_{k}^{j} y_{n}^{m} \text { holds in } M \text { iff } m \leqq k ;
\end{array} \\
f_{1}\left(d_{i}\right)=d_{i-1}, \quad f_{2}\left(d_{i} d_{j}\right)=y_{i-1}^{i}, \quad f_{3}\left(d_{i} d_{j} y_{m}^{k}\right)=y_{0}^{i} .
\end{gathered}
$$

Now, $\quad Z(Y)=A x_{\mathbf{k}}^{r} E x_{j}^{r} A x_{i}^{r} E x_{i}^{m} A x_{j}^{m} E x_{\mathbf{k}}^{m}\left(x_{i}^{m} x_{i}^{r} \wedge\left(x_{j}^{m} x_{j}^{r} \supset x_{k}^{m} x_{k}^{r} \wedge x_{i}^{p} x_{i}^{m} x_{j}^{m} \wedge \sim x_{i}^{p} x_{i}^{m} x_{k}^{m}\right)\right)$, but under the above interpretation,

$$
\begin{aligned}
f_{2}\left(d_{i} d_{j}\right) d_{i} & \wedge\left({ }_{y_{m}}^{k} f_{1}\left(d_{i}\right) \supset f_{3}\left(d_{i} d_{j} y_{j}^{k}\right) d_{i} \wedge{ }_{x_{i} f_{2}}\left(d_{i} d_{j}\right) y_{m}^{k}\right. \\
& \left.\wedge \sim x_{i}^{p} f_{2}\left(d_{i} d_{j}\right) f_{3}\left(d_{i} d_{j} y_{m}^{k}\right)\right)
\end{aligned}
$$

holds in $M$ for any choice of $d_{i}, d_{j}, y_{m}^{k}$, where $f_{1}, f_{2}, f_{3}$ are the functions given above, since

$$
\stackrel{y_{i-1}^{j}}{i} d_{i} \wedge\left({ }_{y_{m}}^{k} d_{i-1} \supset y_{0}^{i} d_{i} \wedge \stackrel{p}{x_{t} y_{i-1}^{j} y_{m}^{k}} \wedge \sim x_{t}^{p} y_{i-1}^{j} y_{0}^{i}\right)
$$


is satisfied in $M$. But it is easily checked that $S_{Z}$ holds in $M$. Thus, $\{Z(Y)\}$ $\cup S_{Z}$ is consistent, but $Y$ is contradictory. We assert now that

5.11. Theorem $Z(X) \wedge Z(Y) \supset Z(\Lambda(X, Y))$ is deducible from $S_{Z}$ provided that $X \in \mathcal{Y}, Y \in \mathcal{Y}$, and variables of the same class are quantified in $X$ and $Y$. We demonstrate the theorem for the case in which neither $X$ nor $Y$ has a prefix of the form

$$
\cdots E \cdots A \cdots E \cdots
$$

since we require the theorem only for this case, where $\Lambda$ for the purpose of this demonstration is restricted to the following syntactical transform which is applied only to wff so described: let

$$
\begin{aligned}
& P=A x_{i}^{m} \cdots A x_{i_{s}}^{m} E x_{i_{1}}^{m} \cdots E x_{i_{t}}^{m} A x_{k_{1}}^{m} \cdots A x_{k_{u}}^{m}(Q), \\
& Q=A x_{p_{1}}^{m} \cdots A x_{p_{f}}^{m} E x_{q_{1}}^{m} \cdots E x_{q_{g}}^{m} A x_{r_{1}}^{m} \cdots A x_{r_{h}}^{m}(B),
\end{aligned}
$$

then

$$
\begin{aligned}
& \Lambda(P, Q)=A x_{i_{1}}^{m} \cdots A x_{i_{\varepsilon}}^{m} A x_{p_{1}}^{m} \cdots A x_{p_{f}}^{m} E x_{j_{1}}^{m} \cdots E x_{j_{t}}^{m} E x_{q_{1}}^{m} \cdots E x_{q_{g}}^{m} A x_{k_{1}}^{m} \cdots \\
& A x_{k_{u}}^{m} A x_{r_{1}}^{m} \cdots A x_{r_{h}}^{m}(a \wedge ß) \text {. }
\end{aligned}
$$

We see that when $\Lambda$ is applied to a pair of wff belonging to $\mathcal{Y}$, it produces a wff belonging to $\mathcal{Y}$ and equivalent to the conjunction of the given wff.

We demonstrate 5.11 by applying repeatedly the following wff; this transform can be generalized so that $\Lambda(x, y)$ is equivalent to $x \wedge y$ and is in prenex normal form.

$$
(a \times b) \wedge c \supset a \times(b \wedge c)
$$

where $X$ stands for $\wedge$ and $\vee$, but not for both simultaneously in the same formula. It is easily seen that 5.12 is provable, since

$$
\begin{aligned}
& (a \vee b) \wedge c \supset(a \vee b) \wedge(a \vee c) \text { is provable, and } \\
& (a \vee b) \wedge(a \vee c) \equiv a \vee(b \wedge c) \text { is provable. }
\end{aligned}
$$

Now, let $a$ be the matrix of $C(X)$, and $\beta$ be the matrix of $C(Y)$. Then $a$ is of the form $a_{1} \times\left(b_{1} \times\left(\cdots \times\left(n_{1} \times P\right) \cdots\right)\right.$, while $B$ is of the form $a_{2} \times\left(b_{2} \times\left(\cdots \times\left(m_{2} \times Q\right) \cdots\right)\right.$, where $\times$ may be $\wedge$ or $\vee$. We apply 5.12 to $Q \wedge ß$; this gives

$$
a_{1} \times\left(\left(b_{1} \times \cdots \times n_{1} \times P\right) \wedge\left(a_{2} \times b_{2} \times \cdots \times m_{2} \times Q\right)\right) .
$$

We now apply 5.12 to $\left(\left(b_{1} \times \cdots \times n_{1} \times P\right) \wedge\left(a_{2} \times b_{2} \times \cdots \times m_{2} \times Q\right)\right)$ removing either $b_{1}$ or $a_{2}$ as we see fit. Continuing this process, we find that

5.13. $a \wedge ß \supset \mathfrak{e} \times P \wedge Q$ is provable, where $\mathcal{C}$ consists of $a_{1}, b_{1}, \cdots, n_{1}$ and $a_{2}, b_{2}, \cdots, m_{2}$ intermingled in any manner provided only that the ordering in $Q$ and $B$ is maintained. But if $B \supset C$ is provable, so are $A x_{i}^{m}(B) \supset A x_{i}^{m}(C)$ and $E x_{i}^{m}(B) \supset E x_{i}^{m}(C)$. Applying this rule to 5.13 we obtain 
5.14. $\Lambda(Z(X), Z(Y)) \supset \cdots(\mathfrak{e} \times P \wedge Q)$ is provable where the implicate differs from $Z(\Lambda(X, Y))$ in that there are surplus quantifiers with superscript $r$ in the prefix, and the matrix contains corresponding relativizing sequences with subscript $j$, say, rather than $i$. It is apparent that unwanted universal quantifiers may be discarded, and the corresponding relativizing sequences replaced as desired, since if

$$
A x_{i}^{r} A x_{j}^{r}\left(x_{i}^{m} x_{i}^{r} \wedge x_{j}^{m} x_{j}^{r} \supset B\right)
$$

holds in a structure, then

$$
A x_{i}^{r}\left(x_{i}^{m} x_{i}^{r} \wedge x_{j}^{m} x_{i}^{r} \supset B\right)
$$

holds in the same structure. However, to eliminate unwanted existential quantifiers we must make use of the set $S_{\boldsymbol{Z}}$; suppose

$$
A x_{k}^{r} E x_{i}^{r} E x_{j}^{r}\left(P \supset x_{t}^{m} x_{i}^{r} \wedge x_{s}^{m} x_{i}^{r} \wedge Q\right) \wedge S_{Z}
$$

holds in a structure. Then there are functions $f\left(x_{k}^{r}\right)$ and $g\left(x_{k}^{r}\right)$ such that $P \supset x_{t}^{m} f\left(x_{k}^{r}\right) \wedge x_{s}^{m} g\left(x_{k}^{r}\right) \wedge Q$ holds in the structure for $k=1,2, \cdots$. We now define the function $h\left(x_{k}^{\tau}\right)$ as follows:

$$
h\left(x_{k}^{r}\right)=\left\{\begin{array}{l}
f\left(x_{k}^{r}\right) \text { if } g\left(x_{k}^{r}\right) f\left(x_{k}^{r}\right) \text { holds } \\
g\left(x_{k}^{r}\right) \text { otherwise. }
\end{array}\right.
$$

It is easily checked that

$$
P \supset x_{t}^{m} h\left(x_{k}^{r}\right) \wedge x_{s}^{m} h\left(\stackrel{r}{x_{k}}\right) \wedge Q
$$

holds in the structure, since $S_{Z}$ holds in the structure. Thus, $A x_{k}^{r} E x_{i}^{r}\left(P \supset x_{t}^{m} x_{i}^{r}\right.$ $\left.\wedge x_{s}^{m} x_{i}^{r} \wedge Q\right)$ holds in the structure. We have now shown that the implicate of 5.14 implies $Z(\Lambda(X, Y))$ is deducible from $S_{Z}$; thus

$$
Z(X) \wedge Z(Y) \supset Z(\Lambda(X, Y))
$$

is deducible from $S_{Z}$.

We wish to show now that 5.8 holds even if $Y$ has fewer changes from $A$ to $E$ and from $E$ to $A$ then has 5.9. Suppose that a wff takes the form

$$
Y=A x_{i}^{m} E x_{j}^{m}(B)
$$

and that $\{Z(Y)\} \cup S_{Z}$ is consistent. But $x_{k}^{m} x_{k}^{r} \supset x_{k}^{m} x_{k}^{r}$ holds in any structure in which it is defined; thus, $E x_{k}^{\tau} A x_{k}^{m}\left(x_{k}^{m} x_{k}^{r} \supset x_{k}^{m} x_{k}^{r}\right)$ holds in any structure satisfying $S_{Z}$; hence, $\{Z(Y)\} \cup\left\{Z\left(A x_{k}^{m}\left(x_{k}^{m} x_{k}^{r}\right)\right)\right\} \cup S_{Z}$ is consistent. But then, by 5.11, $\left\{Z\left(A x_{i}^{m} E x_{j}^{m} A x_{k}^{m}\left(B \wedge x_{k}^{m} x_{k}^{r}\right)\right)\right\} \cup S_{Z}$ is consistent; hence, by 5.8, $A x_{i}^{m} E x_{j}^{m} A x_{k}^{m}\left(B \wedge x_{k}^{m} x_{k}^{r}\right)$ is consistent. Thus, $A x_{i}^{m} E x_{j}^{m}(B)$ is consistent. It is apparent that the other cases follow in a similar manner. 
We wish to show that if the prefix of $Y$ contains $E \cdots A \cdots E$, where the dots represent quantifiers, then 5.8 does not hold in general, i.e. we can find a matrix such that 5.8 is false. Now, $X=E x_{i}^{m} A x_{j}^{m} E x_{k}^{m}\left(x_{i}^{p} x_{i}^{m} x_{j}^{m} \wedge \sim x_{i}^{p} x_{i}^{m} x_{k}^{m}\right)$ is contradictory, while $\{Z(X)\} \cup S_{Z}$ is consistent; also, $E x_{s}^{r} A x_{s}^{m}\left(x_{s}^{m} x_{s}^{r} \supset x_{s}^{m} x_{s}^{r}\right)$ holds in any structure satisfying $S_{z}$. Thus,

$$
\{Z(X)\} \cup\left\{E x_{8}^{r} A x_{s}^{m}\left(x_{8}^{m} x_{8}^{r} \supset x_{8}^{m} x_{8}^{r}\right)\right\} \cup S_{Z}
$$

is consistent; but it is easily shown by the method of 5.11 that $\{Z(Y)\} \cup S_{Z}$ is consistent, where

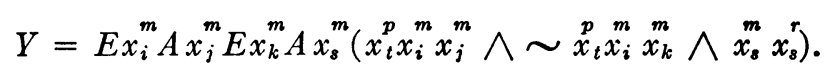

Thus, $Y$ is contradictory while $\{Z(Y)\} \cup S_{Z}$ is consistent. By repeating the process on $Y$ in place of $X$, it is clear that, by placing the quantifier where we will, we can introduce as many universal quantifiers into the prefix as we wish.

We show that we can introduce existential quantifiers where we will by considering

$$
W=X \wedge \operatorname{Ex}_{i}^{m}(a \supset a) .
$$

Here, $W$ is contradictory, but it is easily shown that $\{Z(W)\} \cup S_{Z}$ is consistent.

We now define the syntactical transform $N(Y)$ which applies only to wff belonging to the class $\mathrm{Z}: N(Y)$ is obtained from $Y$ by interchanging $A$ and $E$ everywhere in the prefix of $Y$, and inserting $\sim$ before the matrix of $Y$; thus, $N(Y) \equiv \sim Y$. We have established

5.15. Theorem. $Y$ is consistent provided $\{Z(Y)\} \cup S_{Z}$ is consistent, and $N(Y) \in \mathrm{Z}$.

This theorem is expressed in descriptive or semantic terms; we wish now to express it in terms of provability. Thus, we shall prove

5.16. Theorem. If $Y$ is provable then $Z(Y)$ is deducible from $S_{Z}$, provided $Y \in Z$.

For this purpose we must first establish

5.17. $\sim Z(Y) \equiv Z(N(Y))$ is provable. Let

$$
Y=\cdots A x_{\imath}^{m} \cdots E x_{j}^{m} \cdots
$$

then

$$
\begin{aligned}
Z(Y)=\cdots A x_{i}^{r} \cdots E x_{i}^{r} \cdots A x_{i}^{m} \cdots E x_{j}^{m} \cdots & \\
& \left(\cdots x_{i}^{m} x_{i}^{r} \supset \cdots x_{j}^{m} x_{j}^{r} \wedge \cdots\right.
\end{aligned}
$$

and 
$N(Z(Y)) \equiv \cdots E x_{j}^{r} \cdots A x_{i}^{r} \cdots E x_{i}^{m} \cdots A x_{j}^{m} \cdots$

$$
\left(\cdots x_{i}^{m} x_{i}^{r} \wedge \cdots x_{j}^{m} x_{i}^{r} \supset \cdots \sim a\right)
$$

but

$$
N(Y)=\cdots E x_{i}^{m} \cdots A x_{i}^{m} \cdots(\sim Q)
$$

and

$Z(N(Y))=\cdots E x_{j}^{r} \cdots A x_{i}^{r} \cdots E x_{i}^{m} \cdots A x_{j}^{m} \cdots$

$\left(\cdots x_{i}^{m} x_{i}^{r} \wedge \cdots x_{i}^{m} x_{i}^{r} \supset \cdots \sim a\right)$

hence,

$$
N(Z(Y)) \equiv Z(N(Y)) \text { is provable, }
$$

thus,

$$
\sim Z(Y) \equiv Z(N(Y)) \text { is provable. }
$$

Now let $X$ be any provable wff such that $X \in \mathrm{Z}$, then 5.15 asserts that $N(X)$ is consistent provided that $\{Z(N(X))\} \cup S_{\boldsymbol{Z}}$ is consistent. Hence, we can assert that $\sim Z(N(X))$ holds in any structure which satisfies $S_{Z}$; thus, $\sim Z(N(X))$ is deducible from $S_{Z}$, i.e. $Z(X)$ is deducible from $S_{Z}$. This establishes 5.16 .

Let $X_{1}, \cdots, X_{n}$ be a set of wff in which only one class is quantified, and such that $N\left(X_{i}\right) \in \mathrm{Z}, i=1,2, \cdots, n$. We define the syntactical transform $\Lambda\left(X_{1}, \cdots, X_{n}\right)$ as follows: let $X^{1}=\Lambda\left(X_{1}, X_{2}\right), \cdots, X^{k}=\Lambda\left(X^{k-1}, X_{k+1}\right), \cdots$, $X^{n-1}=\Lambda\left(X^{n-2}, X_{n}\right)$; then $X^{n-1} \equiv X_{1} \wedge \cdots \wedge X_{n}$, and $N\left(X^{n-1}\right) \in Z$. We denote $X^{n-1}$ by $\Lambda\left(X_{1}, \cdots, X_{n}\right)$.

We establish now

5.18. Theorem. $Z\left(X_{1}\right) \wedge \cdots \wedge Z\left(X_{n}\right) \supset Z(Y)$ is deducible from $S_{Z}$ provided $X_{1} \wedge \cdots \wedge X_{n} \supset Y$ is provable, $Y \in Z$, and $N\left(X_{i}\right) \in Z i=1, \cdots, n$.

We have that $N\left(\Lambda\left(\Lambda\left(X_{1}, \cdots, X_{n}\right), N(Y)\right)\right)$ is provable and belongs to the class $\mathrm{Z}$; hence, from $\left.5.16, Z\left(N\left(\Lambda\left(\Lambda X_{1}, \cdots, X_{n}\right), N(Y)\right)\right)\right)$ is deducible from $S_{Z}$; but from 5.11, $Z(X) \wedge Z(N(Y)) \supset Z(\Lambda(X, N(Y)))$ is deducible from $S_{Z}$, if $N(X) \in Z$; thus, by $5.17, Z(N(\Lambda(X, N(Y)))) \supset(Z(X) \supset Z(Y))$ is deducible from $S_{Z}$. Hence, $Z\left(\Lambda\left(X_{1}, \cdots, X_{n}\right)\right) \supset Z(Y)$ is deducible from $S_{Z}$; but from $5.11, Z\left(X_{1}\right) \wedge \cdots \wedge Z\left(X_{n}\right) \supset Z\left(\Lambda\left(X_{1}, \cdots, X_{n}\right)\right)$ is deducible from $S_{Z}$; thus, $Z\left(X_{1}\right) \wedge \cdots \wedge Z\left(X_{n}\right) \supset Z(Y)$ is deducible from $S_{Z}$.

6. The uniform continuity transform. We develop now a transform which generalizes the idea of uniform continuity of a function. A wff is transformed by applying two of the above transforms to it; first, we apply the continuity at zero transform, and then we duplicate with respect to one class. We denote by $U(Y)$ the result of applying this transform to the wff $Y$.

Now, in 3 , we showed that provability is invariant under duplication; 
hence, by virtue of 5.16, if $Y$ is provable and $Y \in \mathrm{Z}$ then $D_{m}(Z(Y))$ is deducible from $D_{m}\left(S_{Z}\right)$. Thus, $U(Y)$ is deducible from $D_{m}\left(S_{Z}\right)$. Hence, a supporting set of the $U$ transform is the following, which we denote by $S_{U}$ :

6.4

$$
\begin{aligned}
& A x_{i}^{r} E x_{j_{1}}^{m} E x_{j_{2}}^{m}\left(x_{j_{1}}^{m} x_{j_{2}}^{m} x_{i}^{r}\right) ; \\
& A x_{i}^{r} A x_{j}^{r}\left(x_{i}^{r} x_{j}^{r} \vee x_{j}^{r} x_{i}^{r}\right) ; \\
& A x_{i}^{r} A x_{j}^{r} A x_{k}^{r}\left(x_{i}^{r} x_{j}^{r} \wedge x_{j}^{r} x_{k}^{r} \supset x_{i}^{r} x_{k}^{r}\right) ; \\
& A x_{i_{1}}^{m} A x_{i_{2}}^{m} A x_{j}^{r} A x_{k}^{r}\left(x_{i_{1}}^{m} x_{i_{2}}^{m} x_{j}^{r} \wedge x_{j}^{r} x_{k}^{r} \supset x_{i_{1}}^{m} x_{i_{2}}^{m} x_{k}^{r}\right) .
\end{aligned}
$$

We have established

6.5. Theorem. If $Y$ is provable then $U(Y)$ is deducible from $S_{U}$ provided $Y \in \mathrm{Z}$.

Suppose that $X_{1} \wedge \cdots \wedge X_{n} \supset Y$ is provable, $Y \in \mathbb{Z}$, and $N\left(X_{i}\right) \in \mathcal{Z}$ $i=1,2, \cdots, n$; then, by 5.18 ,

$$
D_{m}\left(Z\left(X_{1}\right) \wedge \cdots \wedge Z\left(X_{n}\right) \supset Z(Y)\right)
$$

is deducible from $D_{m}\left(S_{Z}\right)$, i.e. $U\left(X_{1}\right) \wedge \cdots \wedge U\left(X_{n}\right) \supset U(Y)$ is deducible from $S_{U}$. We have proved

$$
U\left(X_{1}\right) \wedge \cdots \wedge U\left(X_{n}\right) \supset U(Y)
$$

is deducible from $S_{U}$ provided $X_{1} \wedge \cdots \wedge X_{n} \supset Y$ is provable, and $Y \in Z$ and $N\left(X_{i}\right) \in Z i=1,2, \cdots, n$.

We show now that under a suitable interpretation the $U$ transform does generalize the idea of uniform continuity of a function. Consider

$$
Y=A x_{i}^{m} A x_{j}^{m} E x_{k}^{m}\left(B\left(x_{i}^{m} x_{j}^{m} x_{k}^{m}\right)\right),
$$

then

$$
\begin{gathered}
U(Y)=A x_{k}^{r} E x_{i}^{r} A x_{i_{1}}^{m} A x_{i_{2}}^{m} A x_{j_{1}}^{m} A x_{j_{2}}^{m} E x_{k_{1}}^{m} E x_{k_{2}}^{m}\left(x_{i_{1}}^{m} x_{i_{2}}^{m} x_{i}^{r} \wedge x_{j_{1}}^{m} x_{j_{2}}^{m} x_{i}^{r}\right. \\
\left.\supset x_{k_{1}}^{m} x_{k_{2}}^{m} x_{k}^{r} \wedge B_{m}\left(x_{i_{1}}^{m} x_{i_{2}}^{m} x_{j_{1}}^{m} x_{j_{2}}^{m} x_{k_{1}}^{m} x_{k_{2}}^{m}\right)\right) .
\end{gathered}
$$

For any $i, j, k$ we interpret $x_{i}^{m}$ as complex numbers, $x_{i}^{r}$ as positive numbers, $x_{i}^{m} x_{j}^{m} x_{k}^{r}$ as $\left|x_{i}^{m}-x_{j}^{m}\right|<x_{k}^{r}$, and we define $B_{m}\left(x_{i_{1}}^{m} x_{i_{2}}^{m} x_{j_{1}}^{m} x_{j_{2}}^{m} x_{k_{1}}^{m} x_{k_{2}}^{m}\right)$ as follows: we replace each generating wff that appears in $B$ by the conjunction of two such wff; for example, $Q\left(x_{i}^{m} x_{j}^{m} x_{k}^{m}\right)$ is replaced by $Q\left(x_{i_{1}}^{m} x_{j_{1}}^{m} x_{k_{1}}^{m}\right) \wedge Q\left(x_{i_{2}}^{m} x_{j_{2}}^{m} x_{k_{2}}^{m}\right)$. Thus, if $B$ is a conjunction of generating sequences, then $U(Y)$ expresses the uniform continuity of the function which $Y$ states exists.

7. The topological continuity transform. The supporting set required for the $Z$ transform, see 5, represents essentially a simply-ordered set; we introduce now a transform, $T$, whose supporting set represents a partially ordered set. The transform itself differs from that of 5 only in that a quantifier is 
introduced for each quantifier of the given wff, rather than each block of quantifiers in the given wff, and no two variables have the same relativizing sequence. It should be noted that this departure is not strictly necessary since topological continuity can be adequately expressed by the $Z$ transform in view of 7.8. However, the $T$ transform has the advantage of being a more natural choice to represent topological continuity than the $Z$ transform-the $T$ transform can be described more simply than the $Z$ transform-and as a consequence we can establish without difficulty that

$$
T(X) \wedge T(Y) \supset T(\Lambda(X, Y))
$$

is provable, whereas we could show only that the corresponding wff for the $Z$ transform is deducible from $S_{Z}$.

This transform applies only to wff belonging to the class $\mathcal{Y}$. The transform is as follows:

7.1. Adjoin to the left of the prefix the sequence of quantifiers obtained as follows:

(a) write down the prefix in reverse order, interchanging $A$ and $E$,

(b) change all superscripts to those of another class.

7.2. Relativize with respect to the class quantified in the given wff, the relativizing sequence for each quantified variable being the corresponding variable introduced into the prefix.

For example, the transform of

$$
A x_{i}^{m} A x_{j}^{m} E x_{k}^{m}(B)
$$

is

$$
A x_{k}^{r} E x_{j}^{r} E x_{i}^{r} A x_{i}^{m} A x_{j}^{m} E x_{k}^{m}\left(x_{i}^{m} x_{i}^{r} \wedge x_{j}^{m} x_{j}^{r} \supset x_{k}^{m} x_{k}^{r} \wedge ß\right) .
$$

If in the interpretation the $x_{i}^{m}$ are points and the $x_{i}^{r}$ are neighborhoods of one point, and $x_{i}^{m} x_{j}^{r}$ is interpreted as $x_{i}^{m} \in x_{j}^{r}$, then the transform of the above wff expresses the topological continuity at the fixed point of the function of $x_{i}^{m}$ and $x_{j}^{m}$ which satisfies $B$.

\subsection{THEOREM. If $Y \in Y$ and has the form}

$$
A x_{i_{1}}^{m} \cdots A x_{i_{s}}^{m} E x_{j_{1}}^{m} \cdots E x_{j_{t}}^{m} A x_{k_{1}}^{m} \cdots
$$

$$
A x_{k_{u}}^{m}\left(B\left(x_{i_{1}}^{m} \cdots x_{i_{s}}^{m}, x_{i_{1}}^{m} \cdots x_{i_{t}}^{m}, x_{k_{1}}^{m} \cdots x_{k_{u}}^{m}\right)\right)
$$

then $Y$ is consistent provided $S_{T} \cup\{T(Y)\}$ is consistent, where $S_{T}$ consists of the following:

$$
A x_{i}^{r} E x_{j}^{m}\left(x_{j}^{m} x_{i}^{r}\right) \text {; }
$$

$$
A \stackrel{r}{x_{i}} A \dot{x}_{j}^{r} A \stackrel{x_{k}^{r}}{x_{x}^{r}}\left(x_{i}^{r} \wedge \dot{x}_{j}^{r} x_{k}^{r} \supset x_{i}^{r} x_{k}^{r}\right) \text {; }
$$




$$
A x_{i}^{m} A x_{j}^{r} A x_{k}^{r}\left(x_{i}^{m} x_{j}^{r} \wedge x_{j}^{r} x_{k}^{r} \supset x_{i}^{m} x_{k}^{r}\right)
$$
$A x_{i}^{r} A x_{j}^{r} E x_{k}^{r} A x_{t}^{r}\left(x_{k}^{r} x_{i}^{r} \wedge x_{k}^{r} x_{j}^{r} \wedge\left(x_{t}^{r} x_{i}^{r} \wedge x_{t}^{r} x_{i}^{r} \supset x_{t}^{r} x_{k}^{r}\right)\right) ;$ $A x_{i}^{r}\left(x_{i}^{r} x_{i}^{r}\right)$.

Proof. We suppose we are given a structure $S^{*}$ such that $S_{T} \cup\{T(Y)\}$ holds in $S^{*}$. Consider any set of constants of $S^{*}$ which correspond to variables of the class $r$, say, $y_{1}^{r} \cdots y_{n}^{r}$, which is such that $y_{1}^{r} y_{j}^{r} \wedge y_{j}^{r} y_{1}^{r}$ holds in $S^{*}$ for $j=2,3, \cdots, n$; then we form the structure $S^{* *}$ which is obtained from $S^{*}$ by eliminating all such sets of constants $y_{2}^{r} \cdots y_{n}^{r}$, and retaining all other constants and truth values. Hence, in $S^{* *}$ if $y^{r} z^{r} \wedge z^{r} y^{r}$ holds, then $y^{r}=z^{r}$.

We shall show that $S_{T} \cup\{T(Y)\}$ holds in $S^{* *}$ : clearly, 7.5, 7.6, 7.7, 7.9 hold in $S^{* *}$; consider 7.8. Suppose that for a pair of constants $x^{r}$ and $y^{r}$ of $S^{* *}$ the constant $z^{r}$ which exists in $S^{*}$ by virtue of 7.8 is not in $S^{* *}$. Then there is a $w^{r}$ in $S^{* *}$ such that $w^{r} z^{r} \wedge z^{r} w^{r}$ holds in $S^{*}$; hence, by $7.6, w^{r} x^{r} \wedge w^{r} y^{r}$ holds in $S^{* *}$, and for any $u^{r}, u^{r} x^{r} \wedge u^{r} y^{r} \supset u^{r} w^{r}$ holds in $S^{* *}$. That $T(Y)$ holds in $S^{* *}$ follows from 7.7 , since

$$
x^{r} y^{r} \wedge y^{r} x^{r} \supset\left(a x^{r} \supset a y^{r}\right) \wedge\left(a y^{r} \supset a x^{r}\right)
$$

holds in $S^{*}$; thus, if $x^{r} y^{r} \wedge y^{r} x^{r}$ holds in $S^{*}$, then $A x\left(x x^{r} \equiv x y^{r}\right)$ holds in $S^{*}$.

It is now clear that $T(Y)$ holds in $S^{* *}$ since if some constant $x^{r}$ which satisfies $T(Y)$ in $S^{*}$ does not belong to $S^{* *}$, then there is a constant $y^{r}$ of $S^{* *}$ such that $A x\left(x x^{r} \equiv x y^{r}\right)$ holds in $S^{*}$; hence, the constant $y^{r}$ satisfies $T(Y)$ equally as well as $x^{r}$.

We shall denote the constants of $S^{* *}$ which exist by virtue of 7.8 for each $x^{r}$ and $y^{r}$ in $S^{* *}$ by $x^{r} \cap y^{r}$.

We show that $\left(x^{r} \cap y^{r}\right) \cap z^{r}=x^{r} \cap\left(y^{r} \cap z^{r}\right)$ in $S^{* *}$ : denote $x^{r} \cap y^{r}$ by $u_{1}^{r}$, and $y^{r} \cap z^{r}$ by $u_{2}^{r}$, and $u_{1}^{r} \cap z^{r}$ by $v_{1}^{r}$, and $x^{r} \cap u_{2}^{r}$ by $v_{2}^{r}$. Then $v_{2}^{r} x^{r} \wedge v_{2}^{r} u_{2}^{r}$ holds in $S^{* *}$, but $u_{2}^{r} y^{r}$ holds, hence, $v_{2}^{r} x^{r} \wedge v_{2}^{r} y^{r}$ holds; thus $v_{2}^{r} u_{1}^{r}$ holds in $S^{* *}$. Also, $v_{2}^{r} z^{r}$ holds in $S^{* *}$; hence, $v_{2}^{r} v_{1}^{r}$ holds in $S^{* *}$. Similarly, $v_{1}^{r} v_{2}^{r}$ holds in $S^{* *}$; thus $v_{1}^{r}=v_{2}^{r}$. Thus, we may disregard brackets and write $x^{r} \cap y^{r} \cap z^{r}$. It follows that $y_{1}^{r} \cap \cdots \cap y_{n}^{r}$ is independent of any bracketing, and may be written without brackets.

Now, $T(Y)$ holds in $S^{* *}$, hence

$$
\begin{aligned}
a_{1} h_{1}\left(y_{1}^{r} \cdots y_{t}^{r}\right) & \wedge \cdots \wedge a_{s} h_{s}\left(y_{1}^{r} \cdots y_{t}^{r}\right) \supset f_{1}\left(y_{1}^{r} \cdots y_{t}^{r}, a_{1} \cdots a_{s}\right) y_{1}^{r} \\
& \wedge \cdots \wedge f_{t}\left(y_{1}^{r} \cdots y_{t}^{r}, a_{1} \cdots a_{s}\right) y_{t}^{r} \wedge\left(b_{1} z_{1}^{r} \wedge \cdots \wedge b_{u} z_{u}^{r}\right. \\
& \left.\supset \leftrightarrow\left(a_{1} \cdots a_{s}, f_{1} \cdots f_{t}, b_{1} \cdots b_{u}\right)\right)
\end{aligned}
$$

holds in $S^{* *}$ for any $a_{1} \cdots a_{s}, y_{1}^{\tau} \cdots y_{t}^{\tau}, b_{1} \cdots b_{u}$ where $h_{1} \cdots h_{s}, f_{1} \cdots f_{t}$ are given functions over $S^{* *}$, and $z_{1}^{r} \cdots z_{u}^{r}$ are given constants in $S^{* *}$.

We choose $y_{i}^{r}=y^{r}$ for $i=1,2, \cdots, t$; and define the function $h$ over $S^{* *}$ by $h\left(y^{r}\right)=h_{1}\left(y^{r} \cdots y^{r}\right) \cap \cdots \cap h_{s}\left(y^{r} \cdots y^{r}\right)$; also, let $z^{r}=z_{1}^{r} \cap \cdots \cap z_{u}^{r}$; then we have, by virtue of $7.6,7.7$, and 7.8 , that 
$a_{1} h\left(y^{r}\right) \wedge \cdots \wedge a_{s} h\left(y^{r}\right) \supset f_{1}\left(y^{r}, a_{1} \cdots a_{s}\right) y^{r} \wedge \cdots \wedge f_{t}\left(y^{r}, a_{1} \cdots a_{s}\right) y^{r}$ 7.11 $\wedge\left(b_{1} z^{r} \wedge \cdots \wedge b_{u} z^{r} \supset \otimes\left(a_{1} \cdots a_{s}, f_{1}\left(y^{r}, a_{1} \cdots a_{s}\right) \cdots\right.\right.$

$$
\left.\left.f_{t}\left(y^{r}, a_{1} \cdots a_{s}\right), b_{1} \cdots b_{u}\right)\right)
$$

holds in $S^{* *}$ for any $a_{1} \cdots a_{s}, y^{r}, b_{1} \cdots b_{u}$.

We now construct a structure $S$ in which 7.4 holds. The constants of $S$ are obtained from those of $S^{* *}$ and $t$ symbols $F_{1}\left(x_{1} \cdots x_{s}\right), \cdots, F_{t}\left(x_{1} \cdots x_{s}\right)$ each having $s$ argument places. First, we say that a constant of $S^{* *}$ has order zero in $S$; next, we say that $F_{i}\left(x_{1} \cdots x_{s}\right), i=1,2, \cdots, t$, is a constant of $S$ of order $n$ if its arguments are constants of $S$ of order $<n$, and at least one argument has order $n-1$. We denote the constants of $S$ by

$$
c_{1}, c_{2}, \cdots, c_{n}, \cdots
$$

where $c_{i}$ precedes $c_{j}$ if the order of $c_{i}$ is not greater than the order of $c_{j}$.

We now define two mappings of the constants of $S$ of order $\leqq k$ into those of $S^{* *}$ :

$$
\begin{aligned}
& \mu c_{m}=\left\{\begin{array}{l}
z^{r} \text { if } c_{m} \text { has order } k, \\
\mu F_{1}\left(c_{m} \cdots c_{m}\right) \cap h\left(\mu F_{1}\left(c_{m} \cdots c_{m}\right)\right) \text { otherwise; }
\end{array}\right. \\
& \lambda c_{m}=a_{i} \text { if } c_{m} \text { has order zero, where } a_{i} \mu c_{m} \text { holds in } S^{* *} ; \\
& \lambda F_{i}\left(c_{j_{1}} \cdots c_{j_{s}}\right)=f_{i}\left(\mu F_{1}\left(c_{j_{1}} \cdots c_{j_{s}}\right), \lambda c_{j_{1}} \cdots \lambda c_{j_{s}}\right) .
\end{aligned}
$$

Note that $\mu c_{m} \mu F_{1}\left(c_{m} \cdots c_{m}\right) \wedge \mu c_{m} h\left(\mu F_{1}\left(c_{m} \cdots c_{m}\right)\right)$ holds in $S^{* *}$ if $c_{m}$ has order $<k$.

We show that $\mu c_{m}=\mu c_{n}$ if $c_{m}$ and $c_{n}$ have the same order. True if $c_{m}$ has order $k$; suppose true if $c_{m}$ has order $>j$. Let $c_{p}$ and $c_{q}$ have order $j<k$; then $\mu c_{p} \mu F_{1}\left(c_{p} \cdots c_{p}\right) \wedge \mu c_{p} h\left(\mu F_{1}\left(c_{p} \cdots c_{p}\right)\right)$ holds, but $F_{1}\left(c_{p} \cdots c_{p}\right)$ and $F_{1}\left(c_{q} \cdots c_{q}\right)$ have order $j+1$; hence $\mu F_{1}\left(c_{p} \cdots c_{p}\right)=\mu F_{1}\left(c_{q} \cdots c_{q}\right)$ by the induction assumption; thus, $\mu c_{p} \mu F_{1}\left(c_{q} \cdots c_{q}\right) \wedge \mu c_{p} h\left(\mu F_{1}\left(c_{q} \cdots c_{q}\right)\right)$ holds; hence, by 7.8, $\mu c_{p} \mu c_{q}$ holds. Similarly, $\mu c_{q} \mu c_{p}$ holds; thus, $\mu c_{p}=\mu c_{q}$.

It follows from the above and 7.9 that $\mu c_{m} \mu c_{n}$ holds if order $c_{m} \leqq$ order $c_{n}$; since, $\mu c_{m} \mu F_{1}\left(c_{m} \cdots c_{m}\right)$ holds if $c_{m}$ has order $<k$, i.e. $\mu c_{m} \mu c_{n}$ holds where order $c_{n}=1+\operatorname{order} c_{m} \leqq k$; but $\mu c_{n} \mu c_{p}$ holds if order $c_{p}=1+\operatorname{order} c_{n} \leqq k$; hence, $\mu c_{m} \mu c_{p}$ holds where order $c_{p}=2+\operatorname{order} c_{m} \leqq k$. Continuing this process we see that $\mu c_{m} \mu c_{n}$ holds if order $c_{m} \leqq$ order $c_{n}$.

We show now that $\lambda c_{m} \mu c_{m}$ holds in $S^{* *}$. True if $c_{m}$ has order zero; suppose true if $c_{m}$ has order $<n$. Let $c_{q}=F_{i}\left(c_{j_{1}} \cdots c_{j_{s}}\right)$ have order $n$; then $\lambda c_{j_{1}} \mu c_{j_{1}} \wedge \cdots$ $\wedge \lambda c_{j_{s}} \mu c_{j_{\text {}}}$, holds in $S^{* *}$; hence, by $7.7, \lambda c_{j_{1}} \mu c_{j_{v}} \wedge \cdots \wedge \lambda c_{j_{s}} \mu c_{j_{v}}$, holds, where the order of $c_{j_{\nu}}$ is the maximum order of $c_{j_{1}}, \cdots, c_{j_{e}}$. But $\mu c_{j_{v}} h\left(\mu F_{1}\left(c_{j_{v}} \cdots c_{j_{v}}\right)\right)$ holds, and $\mu F_{1}\left(c_{j_{v}} \cdots c_{j_{v}}\right)=\mu F_{1}\left(c_{j_{1}} \cdots c_{j_{s}}\right)$ hence, $\mu c_{j_{v}} h\left(\mu F_{1}\left(c_{j_{1}} \cdots c_{j_{s}}\right)\right)$ holds; thus, by $7.7, \lambda c_{j_{1}} h\left(\mu F_{1}\left(c_{j_{1}} \cdots c_{j_{s}}\right)\right) \wedge \cdots \wedge \lambda c_{j_{s}} h\left(\mu F_{1}\left(c_{j_{1}} \cdots c_{j_{s}}\right)\right)$ holds in $S^{* *}$. Hence, from 7.11, $f_{i}\left(\mu F_{1}\left(c_{j_{1}} \cdots c_{j_{s}}\right), \lambda c_{j_{1}} \cdots \lambda c_{j_{s}}\right) \mu F_{1}\left(c_{j_{1}} \cdots c_{j_{s}}\right)$ holds in $S^{* *}$; i.e. $\lambda F_{i}\left(c_{j_{1}} \cdots c_{j_{s}}\right) \mu F_{1}\left(c_{j_{1}} \cdots c_{j_{s}}\right)$ holds in $S^{* *}$. But 
$\mu F_{1}\left(c_{j_{1}} \cdots c_{j_{s}}\right) \mu F_{i}\left(c_{j_{1}} \cdots c_{j_{s}}\right)$ holds in $S^{* *}$; thus, by $7.7, \lambda F_{i}\left(c_{j_{1}} \cdots c_{j_{s}}\right)$ $\cdot \mu F_{i}\left(c_{j_{1}} \cdots c_{j_{s}}\right)$ holds in $S^{* *}$, i.e. $\lambda c_{q} \mu c_{q}$ holds in $S^{* *}$.

Now, in 7.11, set $a_{1}=\lambda c_{j_{1}}, \cdots, a_{s}=\lambda c_{j_{s}} ; b_{1}=\lambda c_{k_{1}}, \cdots, b_{u}=\lambda c_{k_{u}} ; y^{r}$ $=\mu F_{1}\left(c_{j_{1}} \cdots c_{j_{0}}\right)$. But from the above we have that

$$
\lambda c_{j_{1}} h\left(\mu F_{1}\left(c_{j_{1}} \cdots c_{j_{s}}\right)\right) \wedge \cdots \wedge \lambda c_{j_{s}} h\left(\mu F_{1}\left(c_{j_{1}} \cdots c_{j_{s}}\right)\right)
$$

holds in $S^{* *}$; also, $\lambda c_{k_{1}} z^{r} \wedge \cdots \wedge \lambda c_{k_{u}} z^{r}$ holds, since $\lambda c_{k_{i}} \mu c_{k_{i}} \wedge \mu c_{k_{i}} z^{r}$ holds. Thus, $B\left(\lambda c_{j_{1}} \cdots \lambda c_{j_{s}}, \lambda F_{1}\left(c_{j_{1}} \cdots c_{j_{s}}\right) \cdots \lambda F_{t}\left(c_{j_{1}} \cdots c_{j_{s}}\right), \lambda c_{k_{1}} \cdots \lambda c_{k_{u}}\right)$ holds in $S^{* *}$.

The argument of 5.8 now establishes that we can define truth values in $S$ so that 7.4 holds in $S$.

We note that the $T$ transform of the counter-example to 5.8 is the same as the $Z$ transform; and that $S_{T}$ holds in the structure $M$ considered there. Thus, the counter-example to 5.8 applies to the $T$ transform as well.

We show now that $T(X) \wedge T(Y) \supset T(\Lambda(X, Y))$ is provable. Let $a$ be the matrix of $T(X)$ and $B$ be the matrix of $T(Y)$. Now, if $P \supset Q$ is provable, so are $A x_{i}^{m}(P) \supset A x_{i}^{m}(Q)$ and $E x_{i}^{m}(P) \supset E x_{i}^{m}(Q)$. Applying this rule to 5.13 we obtain $\Lambda(T(X), \quad T(Y)) \supset T(\Lambda(X, Y))$ is provable. Thus, $T(X) \wedge T(Y)$ $\supset T(\Lambda(X, Y))$ is provable.

As in 5 we can now show that 7.3 holds even if $Y$ has fewer changes from $A$ to $E$ and from $E$ to $A$ then has 7.4 ; and that if the prefix of $Y$ contains $E \cdots A \cdots E$, where the dots represent quantifiers, then we can find a matrix such that 7.3 is false. We now have the theorem

7.12. TheOREM. $Y$ is consistent provided $\{T(Y)\} \cup S_{T}$ is consistent and $N(Y) \in Z$.

Since $T(X) \wedge T(Y) \supset T(\Lambda(X, Y))$ is provable, we have from the proof of 5.18 .

7.13. Theorem. $T\left(X_{1}\right) \wedge \cdots \wedge T\left(X_{n}\right) \supset T(Y)$ is deducible from $S_{T}$ provided that $X_{1} \wedge \cdots \wedge X_{n} \supset Y$ is provable, and $N\left(X_{1}\right) \in \mathrm{Z}, \cdots, N\left(X_{n}\right) \in \mathrm{Z}$, and $Y \in \mathrm{Z}$.

Note. If $Y$ is provable and $Y \in \mathrm{Z}$, then $D_{m}(T(Y))$ is deducible from $D_{m}\left(S_{T}\right)$. Thus, the transform $V$ which first applies the $T$ transform and then applies the $D_{m}$ transform, has for a supporting set 7.6, 7.8, 7.9, 6.1, and 6.4which we denote by $S_{V}$.

From 7.13 we obtain

7.14. Theorem. $V\left(X_{1}\right) \wedge \cdots \wedge V\left(X_{n}\right) \supset V(Y)$ is deducible from $S_{V}$ provided that $X_{1} \wedge \cdots \wedge X_{n} \supset Y$ is provable, and $N\left(X_{1}\right) \in \mathrm{Z}, \cdots, N\left(X_{n}\right) \in \mathrm{Z}$, and $Y \in \mathrm{Z}$.

8. Applications. We wish to show, generally, that if a wff $a$ of a certain type is deducible in a particular algebraic structure, say a group or integral 
domain, then a transform of $a$ holds in another algebraic structure, say an ordered group or a field, as the case may be. Thus, from the existence of a functor in one algebraic structure we pass to the continuity-in a sense made explicit below-of the functor in another algebraic structure.

Example 1. As a first example, we consider a completely divisible, torsion-free abelian group $\left({ }^{2}\right)$. This can be represented by the following axioms. (For " $x_{i}^{1} x_{j}^{1}$," read " $x_{i}^{1}=x_{j}^{1}$ "; for " $x_{i}^{1} x_{j}^{1} x_{k}^{1}$ " read " $x_{i}^{1}+x_{j}^{1}=x_{k}^{1}$ ").

$8.1 A x_{i}^{1}\left(x_{i}^{1} x_{i}^{1}\right)$;

$8.2 A x^{1}: A x_{j}^{1} A x_{k}^{1}\left(x_{i}^{1} x_{j}^{1} \wedge x_{j}^{1} x_{k}^{1} \supset x_{k}^{1} x_{i}^{1}\right)$;

$8.3 A x_{i}^{1} A x_{j}^{1} A x_{k}^{1} A x_{u}^{1} A x_{v}^{1} A x_{w}^{1}\left(x_{i}^{1} x_{j}^{1} x_{k}^{1} \wedge x_{i}^{1} x_{u}^{1} \wedge x_{j}^{1} x_{v}^{1} \wedge x_{k}^{1} x_{w}^{1} \supset x_{u}^{1} x_{v}^{1} x_{w}^{1}\right)$;

$8.4 A x_{i}^{1} A x_{j}^{1} E x_{k}^{1}\left(x_{i}^{1} x_{j}^{1} x_{k}^{1}\right)$;

$8.5 A x_{i}^{1} A x_{j}^{1} E x_{k}^{1}\left(x_{i}^{1} x_{k}^{1} x_{j}^{1}\right)$;

$8.6 A x_{i}^{1} A x_{j}^{1} A x_{k}^{1} A x_{t}^{1} A x_{u}^{1} A x_{v}^{1} A x_{w}^{1}\left(x_{j}^{1} x_{k}^{1} x_{t}^{1} \wedge x_{i}^{1} x_{t}^{1} x_{u}^{1} \wedge x_{i}^{1} x_{j}^{1} x_{v}^{1} \wedge x_{v}^{1} x_{k}^{1} x_{w}^{1} \supset x_{u}^{1} x_{w}^{1}\right)$;

$8.7 A x_{i}^{1} A x_{j}^{1} A x_{k}^{1} A x_{u}^{1}\left(x_{i}^{1} x_{j}^{1} x_{k}^{1} \wedge x_{j}^{1} x_{i}^{1} x_{u}^{1} \supset x_{k}^{1} x_{u}^{1}\right)$;

$8.8\left\{C_{p}\right\} p=2,3,5,7, \cdots$, where $C_{p}$ is

$$
\begin{aligned}
A x_{1}^{1} A x_{2}^{1} \cdots A x_{p}^{1}\left(\sim x _ { 1 } ^ { 1 } x _ { 1 } ^ { 1 } x _ { 1 } ^ { 1 } \supset \left(x_{1}^{1} x_{1}^{1} x_{2}^{1}\right.\right. & \wedge x_{1}^{1} x_{2}^{1} x_{3}^{1} \\
& \left.\left.\wedge \cdots \wedge x_{1}^{1} x_{p-1}^{1} x_{p}^{1} \supset \sim x_{p}^{1} x_{p}^{1} x_{p}^{1}\right)\right) ;
\end{aligned}
$$

$8.9\left\{D_{n}\right\} n=2,3,4, \cdots$, where $D_{n}$ is

$$
A x_{i}^{1} E x_{1}^{1} E x_{2}^{1} \cdots E x_{n}^{1}\left(x_{1}^{1} x_{1}^{1} x_{2}^{1} \wedge x_{1}^{1} x_{2}^{1} x_{3}^{1} \wedge \cdots \wedge x_{1}^{1} x_{n-1}^{1} x_{n}^{1} \wedge x_{i}^{1} x_{n}^{1}\right) \text {. }
$$

From 8.1 and 8.2 we have that $A x_{i}^{1} A x_{j}^{1}\left(x_{i}^{1} x_{j}^{1} \supset x_{j}^{1} x_{i}^{1}\right)$ holds since, from 8.2, we obtain $A x_{i}^{1} A x_{j}^{1}\left(x_{i}^{1} x_{j}^{1} \wedge x_{j}^{1} x_{j}^{1} \supset x_{j}^{1} x_{i}^{1}\right)$; but from $8.1 x_{j}^{1} x_{j}^{1}$ always holds. The result follows. Note that from 8.7 and 8.4 we can deduce that sums are unique, since $x_{i}^{1} x_{j}^{1} x_{k}^{1} \wedge x_{i}^{1} x_{j}^{1} x_{u}^{1} \wedge x_{j}^{1} x_{i}^{1} x_{t}^{1} \supset x_{k}^{1} x_{t}^{1} \wedge x_{u}^{1} x_{i}^{1}$ holds, but $x_{k}^{1} x_{i}^{1} \wedge x_{u}^{1} x_{i}^{1} \supset x_{k}^{1} x_{u}^{1}$ holds; thus $A x_{i}^{1} A{ }_{j}^{1} A x_{k}^{1} A x_{u}^{1}\left(x_{i}^{1} x_{j}^{1} x_{k}^{1} \wedge x_{i}^{1} x_{j}^{1} x_{u}^{1} \supset x_{k}^{1} x_{u}^{1}\right)$ holds.

We wish to show that the $Z$ transforms of 8.1-8.9 hold in any completely divisible, ordered abelian group, $M$, under the following interpretation:

$x_{i}^{2}$ denotes those elements of $M$ such that $0<x_{i}^{2}$ holds;

$x_{i}^{2} x_{j}^{2}$ is interpreted as $x_{i}^{2}<x_{j}^{2}$;

$x_{i}^{1}$ denotes any element of $M$;

$x_{i}^{1} x_{j}^{2}$ is interpreted as $\left|x_{i}^{1}\right|<x_{j}^{2}$, where $\left|x_{i}^{1}\right|=\left\{\begin{array}{l}x_{i}^{1} \text { if } 0<x_{i}^{1}, \\ -x_{i}^{1} \text { otherwise; }\end{array}\right.$

$x_{i}^{1} x_{j}^{1}$ is interpreted as $x_{i}^{1}=x_{j}^{1}$ in $M$;

$x_{i}^{1} x_{j}^{1} x_{k}^{1}$ is interpreted as $x_{k}^{1}=x_{i}^{1}+x_{j}^{1}$ in $M$.

(2) See [5] where the corresponding analysis is carried out for the Bounding transform. 
Since $M$ is an ordered group its elements satisfy the following conditions:

8.10

$$
\begin{aligned}
A x_{i}^{1}\left(x_{i}^{1}\right. & =0 \vee 0<x_{i}^{1} \vee 0<-x_{i}^{1} \\
& \wedge\left(x_{i}^{1}=0 \supset \sim 0<x_{i}^{1} \wedge \sim 0<-x_{i}^{1}\right) \\
& \wedge\left(0<x_{i}^{1} \supset \sim x_{i}^{1}=0 \wedge \sim 0<-x_{i}^{1}\right) \\
& \left.\wedge\left(0<-x_{i}^{1} \supset \sim x_{i}^{1}=0 \wedge \sim 0<x_{i}^{1}\right)\right)
\end{aligned}
$$

where < denotes the ordering relation in $M$. We say that $x_{i}^{1}<x_{j}^{1}$ holds in $M$ iff $0<x_{j}^{1}-x_{i}^{1} \bigvee x_{i}^{1}=x_{j}^{1}$ holds in $M$. Then, from 8.10, $A x_{i}^{1} A x_{j}^{1}\left(x_{i}^{1}<x_{j}^{1} \bigvee x_{j}^{1}<x_{i}^{1}\right)$ holds in $M$, and from $8.11 A x_{i}^{1} A x_{j}^{1} A x_{k}^{1}\left(x_{i}^{1} \prec x_{j}^{1} \wedge x_{j}^{1} \prec x_{k}^{1} \supset x_{i}^{1} \prec x_{k}^{1}\right)$ holds.

We show that

$$
A x_{i}^{1} A x_{j}^{1}\left(\left|x_{i}^{1}+x_{i}^{1}\right| \prec\left|x_{i}^{1}\right|+\left|x_{j}^{1}\right|\right)
$$

holds in $M$. If $0 \prec x_{i}^{1} \wedge 0<x_{j}^{1}$ then $0 \prec x_{i}^{1}+x_{j}^{1}$; thus, $\left|x_{i}^{1}+x_{j}^{1}\right|=x_{i}^{1}+x_{j}^{1}$. Also $\left|x_{i}^{1}\right|+\left|x_{j}^{1}\right|=x_{i}^{1}+x_{j}^{1}$. If $0<x_{i}^{1} \wedge 0<-x_{j}^{1} \wedge 0<x_{i}^{1}+x_{j}^{1}$, then

$$
\left|x_{i}^{1}+x_{j}^{1}\right|=x_{i}^{1}+x_{j}^{1} \text {, and }\left|x_{i}^{1}\right|+\left|x_{j}^{1}\right|=x_{i}^{1}-x_{j}^{1},
$$

but $x_{i}^{1}+x_{j}^{1}<x_{i}^{1}-x_{j}^{1}$ since $x_{i}^{1}-x_{j}^{1}-\left(x_{i}^{1}+x_{j}^{1}\right)=-2 x_{j}^{1}$ and $0<-2 x_{j}^{1}$.

If $0<x_{i}^{1} \wedge 0<-x_{j}^{1} \wedge 0<-x_{i}^{1}-x_{j}^{1}$, then $\left|x_{i}^{1}+x_{j}^{1}\right|=-x_{i}^{1}-x_{j}^{1} ;$ but $x_{i}^{1}-x_{j}^{1}$ $-\left(-x_{i}^{1}-x_{j}^{1}\right)=2 x_{i}^{1}$, and $0<2 x_{i}^{1}$, i.e. $-x_{i}^{1}-x_{j}^{1}<x_{i}^{1}-x_{j}^{1}$; this establishes 8.12.

We show now that the $Z$ transforms of 8.1-8.9 hold in $M$, where $r=2$. First, we observe that if a wff has the form

$$
Y=A x_{1}^{1} A x_{2}^{1} \cdots A x_{n}^{1}\left(B\left(x_{1}^{1} x_{2}^{1} \cdots x_{n}^{1}\right)\right)
$$

then $Y \supset Z(Y)$ is provable, since

$$
\begin{aligned}
A x_{1}^{1} A x_{2}^{1} \cdots A x_{n}^{1}\left(\circledast\left(x_{1}^{1} x_{2}^{1} \cdots x_{n}^{1}\right)\right) & \\
& \supset\left(x_{1}^{1} x_{i}^{2} \wedge x_{2}^{1} x_{j}^{2} \wedge \cdots \wedge x_{n}^{1} x_{k}^{2} \supset \bigotimes\left(x_{1}^{1} x_{2}^{1} \cdots x_{n}^{1}\right)\right)
\end{aligned}
$$

is provable; thus, $Y \supset Z(Y)$ is provable.

We have now to consider the transforms of 8.4, 8.5, and 8.9. We show that the transform of 8.4 holds in $M$ : consider $A x_{k}^{2} E x_{i}^{2} A x_{i}^{1} A x_{j}^{1} E x_{k}^{1}\left(x_{i}^{1} x_{i}^{2} \wedge x_{j}^{1} x_{i}^{2}\right.$ $\left.\supset x_{k}^{1} x_{k}^{2} \wedge x_{i}^{1} x_{j}^{1} x_{k}^{1}\right)$; if we choose $x_{i}^{2}=x_{i}^{2}$ where $x_{i}^{2}+x_{i}^{2}=x_{k}^{2}$ then $\left|x_{i}^{1}+x_{j}^{1}\right|<\left|x_{i}^{1}\right|$ $+\left|x_{j}^{1}\right| \prec x_{i}^{2}+x_{i}^{2}=x_{t}^{2}+x_{i}^{2}=x_{k}^{2}$. We show that the transform of 8.5 holds in $M$ : consider $A x_{k}^{2} E x_{i}^{2} A x_{i}^{1} A x_{j}^{1} E x_{k}^{1}\left(x_{i}^{1} x_{i}^{2} \wedge x_{j}^{1} x_{i}^{2} \supset x_{k}^{1} x_{k}^{2} \wedge x_{i}^{1} x_{k}^{1} x_{j}^{1}\right)$; if we choose $x_{i}^{2}=x_{i}^{2}$ where $x_{t}^{2}+x_{t}^{2}=x_{k}^{2}$ then $\left|x_{j}^{1}-x_{i}^{1}\right|<\left|x_{j}^{1}\right|+\left|x_{i}^{1}\right|<x_{i}^{2}+x_{i}^{2}=x_{t}^{2}+x_{t}^{2}=x_{k}^{2}$. We show that $Z\left(D_{n}\right)$ holds in $M$;

First, we show that $\left|x_{i}^{1}\right|<\left|x_{i}^{1}+x_{i}^{1}\right|$ holds in $M$ for any $x_{i}^{1}$; if $\left|x_{i}^{1}\right|=x_{i}^{1}$ then $\left|x_{i}^{1}+x_{i}^{1}\right|=x_{i}^{1}+x_{i}^{1}$, hence $\left|x_{i}^{1}+x_{i}^{1}\right|-\left|x_{i}^{1}\right|=x_{i}^{1}$ and $0 \prec x_{i}^{1}$; if $\left|x_{i}^{1}\right|=-x_{i}^{1}$ then $\left|x_{i}^{1}+x_{i}^{1}\right|=-x_{i}^{1}-x_{i}^{1}$, hence $\left|x_{i}^{1}+x_{i}^{1}\right|-x_{i}^{1}=-x_{i}^{1}-x_{i}^{1}-\left(-x_{i}^{1}\right)=-x_{i}^{1}$, and $0<-x_{i}^{1}$. It is now clear in fact that $\left|x_{i}^{1}\right|<\left|x_{i}^{1}+x_{i}^{1}+\cdots+x_{i}^{1}\right|$ holds in $M$. Consider 


$$
\begin{gathered}
Z\left(D_{n}\right)=A x_{1}^{2} E x_{i}^{2} \Lambda x_{i}^{1} E x_{1}^{1} \cdots E x_{n}^{1}\left(x _ { i } ^ { 1 } x _ { i } ^ { 2 } \supset \left(x_{1}^{1} x_{1}^{2} \wedge \cdots \wedge x_{n}^{1} x_{1}^{2} \wedge x_{1}^{1} x_{1}^{1} x_{2}^{1}\right.\right. \\
\left.\left.\wedge x_{1}^{1} x_{2}^{1} x_{3}^{1} \wedge \cdots \wedge x_{1}^{1} x_{n-1}^{1} x_{n}^{1} \wedge x_{i}^{1} x_{n}^{1}\right)\right)
\end{gathered}
$$

choose $x_{i}^{2}=x_{k}^{2}$ where $x_{1}^{2}=x_{k}^{2}+x_{k}^{2}+\cdots+x_{k}^{2} n$ times; then

$$
\left|x_{1}^{1}\right| \prec\left|x_{1}^{1}+\underset{n \text { times }}{\ldots}+x_{1}^{1}\right|=x_{i}^{1}<x_{i}^{2}=x_{k}^{2} \prec x_{1}^{2}
$$

also $\left|x_{2}^{1}\right|<\left|x_{1}^{1}+x_{1}^{1}\right|<\left|x_{1}^{1}\right|+\left|x_{1}^{1}\right|<x_{k}^{2}+x_{k}^{2}<x_{1}^{2}$. Similarly, $\left|x_{k}^{1}\right|<\left|x_{1}^{2}\right|$ holds for $k=3,4, \cdots, n$.

It is easily checked that the supporting set, 5.4-5.7, holds in $M$ under the above interpretation. Applying Theorem 5.18, we now have

8.13. Theorem. If $X$ is a wff which holds in any completely divisible, torsion-free, abelian group, then $Z(X)$ holds in any ordered, completely divisible, abelian group under the above interpretation; provided that $X$ belongs to the class Z.

ExAmple 2. We wish to show that the $U$ transforms of 8.1-8.9 hold in a completely divisible, torsion-free, ordered abelian group $M$ under the following interpretation:

$x_{i}^{1}$ denotes any element of $M$;

$x_{i}^{2}$ denotes any element of $M$ such that $0<x_{i}^{2}$;

$x_{i}^{2} x_{j}^{2}$ is interpreted as $x_{i}^{2}<x_{j}^{2}$;

$x_{i}^{1} x_{j}^{1} x_{k}^{2}$ is interpreted as $\left|x_{i}^{1}-x_{j}^{1}\right| \prec x_{k}^{2}$;

$x_{i}^{1} x_{j}^{1}$ is interpreted as $x_{i}^{1}=x_{j}^{1}$;

$x_{i}^{1} x_{j}^{1} x_{k}^{1}$ is interpreted as $x_{k}^{1}=x_{i}^{1}+x_{j}^{1}$.

We show that the $U$ transform of 8.4 holds in $M$ : Consider $A x_{k}^{2} E x_{i}^{2} A x_{i_{1}}^{1}$ - $A x_{i_{2}}^{1} A x_{j_{1}}^{1} A x_{j_{2}}^{1} E x_{k_{1}}^{1} E x_{k_{2}}^{1}\left(x_{i_{1}}^{1} x_{i_{2}}^{1} x_{i}^{2} \wedge x_{j_{1}}^{1} x_{j_{2}}^{1} x_{i}^{2} \supset x_{k_{1}}^{1} x_{k_{2}}^{1} x_{k}^{2} \wedge x_{i_{1}}^{1} x_{j_{1}}^{1} x_{k_{1}}^{1} \wedge x_{i_{2}}^{1} x_{j_{2}}^{1} x_{k_{2}}^{1}\right)$; choose $x_{i}^{2}=x_{t}^{2}$ where $x_{t}^{2}+x_{t}^{2}=x_{k}^{2}$; then

$$
\begin{aligned}
\left|x_{i_{1}}^{1}+x_{j_{1}}^{1}-\left(x_{i_{2}}^{1}+x_{j_{2}}^{1}\right)\right| & \prec\left|x_{i_{1}}^{1}-x_{i_{2}}^{1}\right|+\left|x_{j_{2}}^{1}-x_{i_{1}}^{1}\right|<x_{i}^{2}+x_{i}^{2} \\
& \prec x_{t}^{2}+x_{t}^{2}<x_{k}^{2} .
\end{aligned}
$$

The $U$ transform of 8.5 can be shown to hold in $M$ in a similar fashion. It is easily checked that the transforms of the other group axioms hold in $M$, and that the supporting set, $S_{U}$, holds in $M$. Applying 6.5 we obtain

8.14. TheOREM. If $X$ holds in every completely divisible, torsion-free, abelian group, then $U(X)$ holds in any ordered, completely divisible, abelian group under the above interpretation; provided that $X \in \mathrm{Z}$.

ExAmple 3. We obtain an integrally closed integral domain of characteristic zero by adjoining to $8.1-8.8$ the following: 
$8.15 A x_{i}^{1} A x_{j}^{1} A x_{k}^{1} A x_{u}^{1} A x_{v}^{1} A x_{v}^{1}\left(x_{1}^{3} x_{i}^{1} x_{j}^{1} x_{k}^{1} \wedge x_{i}^{1} x_{u}^{1} \wedge x_{j}^{1} x_{v}^{1} \wedge x_{k}^{1} x_{w}^{1} \supset x_{1}^{3} x_{u}^{1} x_{v}^{1} x_{w}^{1}\right) ;$

$8.16 A x_{i}^{1} A x_{j}^{1} E x_{k}^{1}\left(x_{1}^{3} x_{i}^{1} x_{j}^{1} x_{k}^{1}\right)$;

$8.17 A x_{i}^{1} A x_{j}^{1} A x_{k}^{1} A x_{t}^{1} A x_{u}^{1} A x_{v}^{1} A x_{w}^{1}\left(x_{1}^{3} x_{j}^{1} x_{k}^{1} x_{t}^{1} \wedge x_{1}^{3} x_{i}^{1} x_{t}^{1} x_{u}^{1} \wedge x_{1}^{3} x_{i}^{1} x_{j}^{1} x_{v}^{1}\right.$

$8.18 A x_{i}^{1} A x_{j}^{1} A x_{k}^{1} A x_{u}^{1}\left(x_{1}^{3} x_{i}^{1} x_{j}^{1} x_{k}^{1} \wedge \stackrel{3}{x_{1} x_{j}^{1} x_{i}^{1} x_{u}^{1}} \supset x_{k}^{1} x_{u}^{1}\right)$;

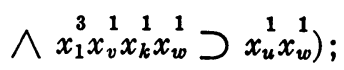

$8.19 A x_{i}^{1} A x_{j}^{1} A x_{k}^{1} A x_{s}^{1} A x_{t}^{1} A x_{u}^{1} A x_{v}^{1} A x_{w}^{1}\left(x_{j}^{1} x_{k}^{1} x_{8}^{1} \wedge x_{1}^{3} x_{i}^{1} x_{8}^{1} x_{t}^{1} \wedge x_{1}^{3} x_{i}^{1} x_{j}^{1} x_{u}^{1}\right.$

$\left\{X_{n}\right\} n=2,3,4, \cdots$, where $X_{n}$ is

$\left.\wedge x_{1}^{3} x_{i}^{1} x_{k}^{1} x_{v}^{1} \wedge x_{u}^{1} x_{v}^{1} x_{w}^{1} \supset x_{t}^{1} x_{w}^{1}\right)$

$A x_{1}^{1} \cdots A x_{n}^{1} E x_{i_{1}}^{1} \cdots E x_{i_{n}}^{1} E x_{j_{1}}^{1} \cdots E x_{i_{n-1}}^{1} E x_{k_{1}}^{1} \cdots E x_{k_{n-2}}^{1} x_{1_{1}}^{1} x_{i_{1}}^{1} x_{i_{1}}^{1} x_{i_{2}}^{1} \wedge \cdots$

$$
\begin{aligned}
& \wedge x_{1}^{3} x_{i_{1}}^{1} x_{i_{n-1}}^{1} x_{i_{n}}^{1} \wedge x_{1}^{3} x_{1}^{1} x_{i_{n-1}}^{1} x_{j_{1}}^{1} \wedge \cdots \wedge x_{1}^{3} x_{n-1}^{1} x_{i_{1}}^{1} x_{j_{n-1}}^{1} \wedge x_{i_{n}}^{1} x_{j_{1}}^{1} x_{k_{1}} \\
& \left.\wedge x_{k_{1}}^{1} x_{j_{2}}^{1} x_{k_{2}}^{1} \wedge \cdots \wedge x_{k_{n-3}}^{1} x_{j_{n-2}}^{1} x_{k_{n-2}}^{1} \wedge x_{k_{n-2}}^{1} x_{i_{n-2}}^{1} x_{n}^{1}\right)
\end{aligned}
$$

$8.21 A x_{i}^{1} A x_{j}^{1} A x_{k}^{1}\left(x_{1}^{3} x_{i}^{1} x_{j}^{1} x_{k}^{1} \wedge x_{k}^{1} x_{k}^{1} x_{k}^{1} \supset x_{i}^{1} x_{i}^{1} x_{i}^{1} \vee x_{j}^{1} x_{j}^{1} x_{j}^{1}\right)$.

In the interpretation,

$x_{1}^{3} x_{i}^{1} x_{j}^{1} x_{k}^{1}$ is read as $x_{k}^{1}=x_{i}^{1} \cdot x_{j}^{1}$;

$x_{i}^{1}$ is interpreted as a complex number, for any $i$;

$x_{i}^{2}$ is interpreted as a positive number, for any $i$;

$x_{i}^{1} x_{j}^{2}$ is read as $\left|x_{i}^{1}\right| \leqq x_{j}^{2}$;

$x_{i}^{1} x_{j}^{1}$ is read as $x_{i}^{1}=x_{j}^{1}$;

$x_{i}^{1} x_{j}^{1} x_{k}^{1}$ is read as $x_{k}^{1}=x_{i}^{1}+x_{j}^{1}$;

Note that from 8.16 and 8.18 we can deduce that products are unique. We do not postulate the existence of a unit element. Note 8.20 asserts that every monic polynomial has a root.

It is easily checked that the supporting set $S_{Z}$ and the $Z$ transforms of 8.1-8.8 and of 8.15-8.21 hold in the field of complex numbers under the above interpretation. Applying 5.18 we obtain

8.22. ThEOREM. If $X$ is a wff which holds in any integrally closed integral domain of characteristic zero, then $Z(X)$ holds in the field of complex numbers under the above interpretation; provided that $X \in \mathrm{Z}$.

A special case of Theorem 8.22 is

8.23. THEOREM. If a system of equations with integral coefficients and with parameters from an integrally closed integral domain of characteristic zero, has a solution in all such integral domains, including integral domains without a unit element, then the solution is continuous in the sense of 8.22 ; provided that the system of equations can be expressed as a wff belonging to the class $\mathrm{Z}$.

EXAMPLE 4. We wish to show now that the $T$ transforms of the group axioms, 8.4-8 6, hold in a structure whose elements on the one hand form a 
group, and on the other hand form a $T_{0}$ space such that there is a neighbourhood which is contained in all other neighbourhoods of the space. Further, we require that the group operations be continuous in the $T_{0}$ space.

We interpret $x_{i}^{1}$ as an element of the group and a point of the topology; $x_{i}^{2}$ is interpreted as a neighbourhood in the topology;

$x_{i}^{2} x_{j}^{2}$ is interpreted as $x_{i}^{2} \subset x_{j}^{2}$ - the neighbourhood $x_{i}^{2}$ is contained in the neighbourhood $x_{j}^{2}$;

$x_{i}^{1} x_{j}^{2}$ is interpreted as $x_{i}^{1} \in x_{j}^{2}$ - the point $x_{i}^{1}$ belongs to the neighbourhood $x_{j}^{2}$;

$x_{i}^{1} x_{j}^{1}$ is interpreted as $x_{i}^{1}=x_{j}^{1}$;

$x_{i}^{1} x_{j}^{1} x_{k}^{1}$ is interpreted as $x_{k}^{1}=x_{i}^{1}+x_{j}^{1}$.

It is easily checked that $S_{T}$ holds in the structure under the above interpretation. We consider the $T$ transform of 8.4 :

$$
A x_{k}^{2} E x_{j}^{2} E x_{i}^{2} A x_{i}^{1} A x_{j}^{1} E x_{k}^{1}\left(x_{i}^{1} x_{i}^{2} \wedge x_{j}^{1} x_{j}^{2} \supset x_{k}^{1} x_{k}^{2} \wedge x_{i}^{1} x_{j}^{1} x_{k}^{1}\right) \text {. }
$$

But this is precisely the statement that addition is continuous in the $T_{0}$ space. The transform of 8.5 is

$$
A x_{k}^{2} E x_{j}^{2} E x_{i}^{2} A x_{i}^{1} A x_{j}^{1} E x_{k}^{1}\left(x_{i}^{1} x_{i}^{2} \wedge x_{j}^{1} x_{j}^{2} \supset x_{k}^{1} x_{k}^{2} \wedge x_{i}^{1} x_{k}^{1} x_{j}^{1}\right),
$$

which is precisely the statement that subtraction is continuous in the $T_{0}$ space.

Applying Theorem 7.13 we have established

8.24. THEOREM. If $Y$ holds in all groups then $T(Y)$ holds in the above structure, provided $Y \in Z$.

EXAMPLE 5. We establish now

8.25. THEOREM. If $Y$ holds in all groups then $T(Y)$ holds in any topological group, provided $Y \in \mathrm{Z}$, where $x_{i}^{2}$ is interpreted as a neighbourhood of the group zero for $i=1,2,3, \cdots$, and the other symbols are interpreted as in the previous example.

Proof. We have only to show that $S_{T}$ and the $T$ transforms of the group axioms hold in a topological group under the above interpretation. It is easily checked that $S_{T}$ and the transform of 8.6 hold in a topological group. We show that the transforms of 8.4 and 8.5 hold also. Now the following conditions hold in a topological group:

(a) if $a$ and $b$ are any two elements of the group then for every neighbourhood $W$ of the element $a+b$ there exist neighbourhoods $U$ and $V$ of the elements $a$ and $b$ such that

$$
x \in U \wedge y \in V \supset x+y \in W,
$$

(b) if $a$ and $b$ are any two elements of the group then for every neighbourhood $W$ of the element $a-b$ there exist neighbourhoods $U$ and $V$ of the elements $a$ and $b$ such that 


$$
x \in U \wedge y \in V \supset x-y \in W .
$$

Hence, from (a) choosing $a=b=0$ we have that for every neighbourhood $W$ of zero there exist neighbourhoods of zero, $U$ and $V$, such that

$$
x \in U \wedge y \in V \supset x+y \in W
$$

and from (b) choosing $a=b=0$ we have that for every neighbourhood $W$ of zero there exist neighbourhoods of zero, $U$ and $V$, such that

$$
x \in U \wedge y \in V \supset x-y \in W
$$

i.e.

$$
A x_{k}^{2} E x_{j}^{2} E x_{i}^{2} A x_{i}^{1} A x_{j}^{1} E x_{k}^{1}\left(x_{i}^{1} x_{i}^{2} \wedge x_{j}^{1} x_{j}^{2} \supset x_{k}^{1} x_{k}^{2} \wedge x_{i}^{1} x_{j}^{1} x_{k}^{1}\right)
$$

and

$$
A x_{k}^{2} E x_{j}^{2} E x_{i}^{2} A x_{i}^{1} A x_{j}^{1} E x_{k}^{1}\left(x_{i}^{1} x_{i}^{2} \wedge x_{j}^{1} x_{j}^{2} \supset x_{k}^{1} x_{k}^{2} \wedge x_{i}^{1} x_{k}^{1} x_{j}^{1}\right)
$$

both hold in the topological group. 8.25 now follows from 7.13.

\section{REFERENCES}

1. J. Herbrand, Recherches sur la théorie de la demonstration, Travaux de la Société des Sciences et des Lettres de Varsovie, Class III, Nr. 33, 1930.

2. D. Hilbert and W. Ackermann, Principles of mathematical logic (English translation), Chelsea, 1950.

3. A. Robinson, Note on an embedding theorem for algebraic systems, J. London Math. Soc. vol. 30 (1955) pp. 249-252.

4. ——, Theorie métamathématique des idéaux, Paris, 1955.

5. Complete theories, Studies in Logic and the Foundations of Mathematics, Amsterdam, 1956.

6. A. Schmidt, Über deduktive Theorien mit mehreren Sorten von Grunddingen, Math. Ann. vol. 115 (1938) pp. 485-506.

7. - Die Zulässigkeit der Behandlung mehrsortigen Theorien mittels der ïblichen einsortigen Prädikatenlogik, Math. Ann. vol. 123 (1946) pp. 187-200.

UNIVERSITY OF AlberTa,

Edmonton, Alta., Canada

UNIVERSITY OF TORONTO,

Toronto, Ont., Canada 